Article

\title{
Characterization of Botanical and Geographical Origin of Corsican "Spring" Honeys by Melissopalynological and Volatile Analysis
}

\author{
Yin Yang, Marie-José Battesti, Jean Costa and Julien Paolini * \\ Laboratory of Natural Product Chemistry, UMR CNRS 6134, Grimaldi Campus, Corsican University, \\ BP 52, Corte 20250, France; E-Mails: yang@univ-corse.fr (Y.Y.); mjbattesti@univ-corse.fr (M.-J.B.); \\ costa@univ-corse.fr (J.C.)
}

* Author to whom correspondence should be addressed; E-Mail: paolini@univ-corse.fr; Tel.: +33-495-450-187; Fax: +33-495-450-257.

Received: 30 September 2013; in revised form: 17 December 2013 / Accepted: 13 January 2014 / Published: 27 January 2014

\begin{abstract}
Pollen spectrum, physicochemical parameters and volatile fraction of Corsican "spring" honeys were investigated with the aim of developing a multidisciplinary method for the qualification of honeys in which nectar resources are under-represented in the pollen spectrum. Forty-one Corsican "spring" honeys were certified by melissopalynological analysis using directory and biogeographical origin of 50 representative taxa. Two groups of honeys were distinguished according to the botanical origin of samples: "clementine" honeys characterized by the association of cultivated species from oriental plain and other "spring" honeys dominated by wild herbaceous taxa from the ruderal and/or maquis area. The main compounds of the "spring" honey volatile fraction were phenylacetaldehyde, benzaldehyde and methyl-benzene. The volatile composition of "clementine" honeys was also characterized by three lilac aldehyde isomers. Statistical analysis of melissopalynological, physicochemical and volatile data showed that the presence of Citrus pollen in "clementine" honeys was positively correlated with the amount of linalool derivatives and methyl anthranilate. Otherwise, the other "spring" honeys were characterized by complex nectariferous species associations and the content of phenylacetaldehyde and methyl syringate.
\end{abstract}

Keywords: honey; clementine and asphodel; melissopalynological analysis; HS-SPME; GC 


\section{Introduction}

The specificity of Corsican honeys is linked with the environmental characteristics of the island (biodiversity of flora, bioclimatic conditions and topography), the endemic black honeybee and typical hive management. Organoleptic and melissopalynological analysis have permitted Corsican honeys to be classified into six ranges: "spring", "spring maquis", "honeydew maquis", "chestnut grove", "summer maquis" and "autumn maquis", according to the harvest season and the geographic location of the apiaries [1]. These honeys have been certified by two official designations of origin: the national Appellation d'Origine Contrôlée (AOC) and the European Protected Designation of Origin (PDO), both marketed as "Miel de Corse-Mele di Corsica" [2,3].

The organoleptic properties of the "spring" honey range are a light color (the lightest among the six ranges) associated with low-to-medium olfactory and aromatic intensities, sometimes with a slight acidity [1-3]. These honeys are described in terms such as floral, fresh fruit, or dry vegetal according to the vocabulary of odor and the aroma wheel [4]. Moreover, the physicochemical characteristics of "spring" honeys are low values of coloration and electrical conductivity. Finally, these honeys are harvested from April to May at low altitudes (below $400 \mathrm{~m}$ ) on the coast, plains or valleys [1-3].

The Corsican "spring" honeys can be classified into two categories. First, honeys harvested in the oriental plain of the island. These cultivated zones are dominated by clementine orchards (Citrus sinensis $\times$ reticulata) associated with other Citrus species, Actinidia sinensis and various fruit trees. They are always surrounded by maquis; an evergreen scrub of vegetation from Mediterranean area. Second, honeys collected in ruderal and/or littoral maquis areas for their first flowering. Ruderal zones are characterized by herbaceous plants, especially Asphodelus ramosus subsp. ramosus (syn: A. microcarpus Salz et Viv.) associated with various species of Fabaceae, Boraginaceae, wild Brassicaceae, Apiaceae and Asteraceae. The coastal areas also showed a diversity of nectariferous and polleniferous resources [5].

Unifloral honeys from the Citrus genus, produced principally from oranges or lemons, are often found in the Mediterranean region (Italy, Spain, Greece, France and North Africa), but also in Israel, USA, Brazil and Mexico [6,7]. The nectar of Asphodelus species is frequently found in the composition of honeys from Mediterranean regions (Italy, Sicily, Corsica and Sardinia), but asphodel unifloral honey is produced mainly in Sardinia [8,9]. In Corsica, the Asphodelus genus is represented by three species: A. ramosus subsp. ramosus, A. cerasiferus and A. fistulosus [10]. A. ramosus subsp. ramosus, which flowers from March to May, was the more visited species.

The certification of geographical and botanical origins of Corsican honeys is conventionally based on the melissopalynological analysis of the entire pollen spectrum [5,11]. Furthermore, sensory characteristics and physicochemical parameters are also necessary to specify the botanical origin of honey [5,11,12]. However, this traditional approach is not precise enough to determine the predominant botanical origin exactly, especially when nectar resources are under-represented in the pollen spectrum. For this reason, the chemical composition of honeys has been used to complete the classical approaches of botanical origin determination. Thus, various extraction methods, such as headspace solid-phase microextraction (HS-SPME), simultaneous steam distillation-solvent extraction and ultrasound-assisted extraction associated with gas chromatography (GC) have been developed for the analysis of the volatile fraction of honeys [13]. Some volatile components, including methyl 
anthranilate, lilac aldehyde and $p$-menth-1-en-9-al, were therefore suggested as the chemical markers of citrus (species not specified) unifloral honey [13-15]. Moreover, Alissandrakis et al. [16] showed that the volatile fractions of citrus flowers (four species) and the corresponding honeys were dominated by linalool derivatives. The phenolic compound hesperetin was also proposed as a botanical indicator of Spanish citrus honeys for its high levels in nectar and honey [17]. Methyl syringate and/or phenylacetaldehyde were identified as characteristic components of nectar from A. microcarpus Salz et Viv. and corresponding unifloral honeys [18,19].

Several techniques (HS-SPME, infrared spectroscopy and ${ }^{1} \mathrm{H}$-nuclear magnetic resonance spectroscopy) have been used to distinguish Corsican and non-Corsican honeys, but these studies did not provide results for the differentiation of the botanical origin of different ranges of Corsican honey [20-22].

According to the geographical and botanical origins of Corsican "spring" honeys certified by melissopalynological analysis, the chemical composition of volatile fractions of honey samples was established using HS-SPME, GC and GC/mass spectrometry (MS). The aim of the study is to establish for the first time a multidisciplinary method for the qualification of Corsican "spring" honeys, based on relationships between the pollen spectrum, volatile chemical markers and some physicochemical parameters.

\section{Experimental Section}

\subsection{Honey and Flower Sampling}

In total, 41 Corsican "spring" honeys (samples 1-41) were selected from our reference bank of honey with AOC and PDO appellations. All these samples were directly packaged in a sealed pot and stored below $14{ }^{\circ} \mathrm{C}$ according to the optimal conditions of honey conservation indicated by Gonnet et al. [23]. The honey samples of three years of harvest (2004-2006) collected in April to June were provided from 12 Corsican producers. The apiaries were located from littoral to $400 \mathrm{~m}$ (principally under $100 \mathrm{~m}$ ) in the oriental cultivated plain or in ruderal and/or maquis zone of thermo- and meso-Mediterranean levels. Clementine (Citrus sinensis $\times$ reticulate, six samples) and Asphodel (Asphodelus ramosus subsp. ramosus, six sample locations) flower specimens were collected in March-May 2009-2012. The nectar secretion during harvest period was ensured by the observation of foraging nectar by honeybees. Flowers samples were analyzed within $48 \mathrm{~h}$.

\subsection{Melissopalynological Analysis}

In this study, melissopalynological analysis was performed using the method described by Yang et al. [24]. Identification of pollen in the "spring" honey was based on the comparison with laboratory's own reference pollen-slides library and also carried out with the palynological expertise practice [5,11] developed for the characterization and the AOC and PDO control of Corsican honeys. Pollen analysis was allowed to establish a total pollen spectrum (qualitative analysis) and pollen density (quantitative analysis) for each honey sample. The identified taxa in the pollen spectrum were expressed in term of relative frequency (RF) and the pollen density was expressed as the absolute number of pollen grain in $10 \mathrm{~g}$ of honey $(\mathrm{PG} / 10 \mathrm{~g})$. 


\subsection{Physicochemical Analysis}

According to the description of Corsican honeys [1,5], two physicochemical parameters, coloration and electrical conductivity were chosen to complete the botanical origin characterization of Corsican "spring" honey. The honey coloration was measured using a Lovibond Comparator apparatus [25]. Results were expressed as millimeters (mm) Pfund. Electrical conductivity was measured at $20{ }^{\circ} \mathrm{C}$ with a conductivity meter micro CM2210 (CRISON, Spain) following the method described by Bogdanov [26] and expressed as milliSiemens per centimeter $(\mathrm{mS} / \mathrm{cm})$.

\subsection{HS-SPME Extraction}

Volatile fractions of honey and flower samples were extracted by HS-SPME with a divinylbenzene/carboxen/polydimethylsiloxane (DVB/CAR/PDMS, $30 \mu \mathrm{m}$ ) fiber (Supelco Sigma Aldrich). The optimization of HS-SPME parameters was performed using two honey samples (9 and 24) and two flower samples (clementine and asphodel flowers). These samples and subsequent analyses (all honey and flower samples studies) were performed in triplicate to ensure that the coefficient of variation ( $\mathrm{CV}$ : ratio of standard deviation to the mean) of the major compounds and the sum of the total peak areas were always $<15 \%$. The samples analyzed were placed in a $20 \mathrm{~mL}$ vial. The parameter optimization was based on the sum of the total peak areas measured using a gas chromatography-flame ionization detection (GC-FID) system. For each sample (both honeys and flowers): the temperatures $\left(25^{\circ} \mathrm{C}, 50{ }^{\circ} \mathrm{C}\right.$ and $70{ }^{\circ} \mathrm{C}$ ), the equilibration times (30, 60 and $\left.90 \mathrm{~min}\right)$ and the extraction times $(15,30$ and $45 \mathrm{~min}$ ) were tested in various experiments. The honey concentration in distilled water was optimized after six different experiments $(0.5 \mathrm{~g} / \mathrm{mL}, 1 \mathrm{~g} / \mathrm{mL}, 1.5 \mathrm{~g} / \mathrm{mL}$ and $2 \mathrm{~g} / \mathrm{mL})$ with $\mathrm{Na}_{2} \mathrm{SO}_{4}$ addition ( $1 \mathrm{~g}$ and $2 \mathrm{~g}$ ). The maximum sum of the total peak areas was obtained from $4 \mathrm{~g}$ of honey sample with $4 \mathrm{~mL}$ of water and $2 \mathrm{~g}$ of $\mathrm{Na}_{2} \mathrm{SO}_{4}$ at a temperature of $70{ }^{\circ} \mathrm{C}$, an equilibrium time of $90 \mathrm{~min}$, and an extraction time of $30 \mathrm{~min}$. The flower weight was optimized after three different experiments ( $1 \mathrm{~g}, 3 \mathrm{~g}$ and $5 \mathrm{~g}$ ). For the Asphodel flowers, the maximum sum of the total peak areas was obtained from $3 \mathrm{~g}$ of sample at a temperature of $70{ }^{\circ} \mathrm{C}$, an equilibrium time of $90 \mathrm{~min}$, and an extraction time of $30 \mathrm{~min}$. Otherwise, the best sampling conditions of Clementine flowers were $1 \mathrm{~g}$ of sample at room temperature $\left(25^{\circ} \mathrm{C}\right)$ with an extraction time of $15 \mathrm{~min}$. Before sampling, the fiber was reconditioned for $5 \mathrm{~min}$ in the $\mathrm{GC}$ injection port at $280{ }^{\circ} \mathrm{C}$. After sampling, the SPME fiber was consecutively inserted into the GC-FID and GC-MS injection ports for $5 \mathrm{~min}$ for desorption of volatile components, both techniques using the splitless injection mode.

\subsection{GC-FID and GC-MS Analysis}

GC-FID analyses were performed using a PerkinElmer (Waltham, MA, USA) AutoSystem XL GC apparatus equipped with a FID system and a fused-silica capillary column $(30 \mathrm{~m} \times 0.25 \mathrm{~mm}$, film thickness $1 \mu \mathrm{m}$ ) coated with Rtx-1 (PDMS). The oven temperature was programmed from 60 to $230{ }^{\circ} \mathrm{C}$ at $2{ }^{\circ} \mathrm{C} / \mathrm{min}$ and then held isothermally at $230{ }^{\circ} \mathrm{C}$ for $35 \mathrm{~min}$. The injector and detector temperatures were maintained at $280{ }^{\circ} \mathrm{C}$. The samples were injected with an SPME inlet liner ( $0.75 \mathrm{~mm}$ i.d.; Supelco) using hydrogen as the carrier gas $(1 \mathrm{~mL} / \mathrm{min})$. The retention indices of the compounds were determined relative to the retention times of a series of $n$-alkanes $\left(\mathrm{C}_{5}-\mathrm{C}_{30}\right)$ with linear 
interpolation. The relative concentrations of components were calculated from the GC peak areas without using correction factors. Samples were also analyzed with a PerkinElmer TurboMass detector (quadrupole), coupled to a GC PerkinElmer AutoSystem XL, equipped with a fused-silica Rtx-1 capillary column. The ion source temperature was $150{ }^{\circ} \mathrm{C}$, and the ionization energy was $70 \mathrm{eV}$. Electronic ionisation (EI) mass spectra were acquired over the mass range of 35-350 Da (scan time $1 \mathrm{~s})$. Other GC conditions were the same as described for the GC-FID analysis. Identification of the components was based on: (1) the comparison of their GC retention indices (RI) on a nonpolar column, determined relative to the retention time of a series of $n$-alkanes with linear interpolation to the retention times of authentic compounds or data with the laboratory's library; (2) the comparison of the RI and spectra with commercial mass spectra libraries [27,28].

\subsection{Statistical Analysis}

The statistical analysis of melissopalynological data was carrying out the methodology previously described by Battesti et al. [11]. In the case of "spring" honey, the inclusion of Citrus and Asphodelus pollen during the nectar foraging is low or very low because of pollen maturity or floral morphology. The "under-representation" of these pollen types and entire pollen spectrum were taken into account for the characterization and comparison of pollen spectrum from "spring" honeys. Principal component analysis (PCA) was carried out using the "PCA" function and canonical correspondence analysis (CCA) was performed with "CCA" function from R software (R Foundation-Institute for Statistics and Mathematics, Austria). CCA is a multidimensional exploratory statistical method in order to demonstrate the correlation between two sets of variables obtained from the same individual.

\section{Results and Discussion}

\subsection{Determination of Geographical and Botanical Origins of Corsican "Spring” Honeys}

The analysis of 41 Corsican "spring" honeys allowed the determination of 92 taxa, including 64 nectariferous taxa and 28 only-polleniferous taxa (Table 1). A biogeographical analysis (biogeographical code: BC [5]) showed the diversity of biogeographical origins of these taxa. Mediterranean species (28 taxa, BC 1-3) associated with Eurasian and Atlantic species (13 taxa, BC 5-6) were well represented in the pollen spectrum. Additionally, cultivated species (four taxa, BC 99) were reported in more than $40 \%$ of honey samples. This distribution was consistent with the database of the characterization of the Corsican honey taxa directory [5,11].

To define the most representative taxa of Corsican "spring" honey, the presence ratio (PR) and the relative frequency (RF) distributions (mean, minimum, maximum, standard deviation and coefficient variation) of each taxon were reported. The pollen directory showed that 50 taxa (T1-T50) could be considered as regionally characteristic species of Corsican "spring" honey for their significant PR $(>10 \%)$ and/or $\mathrm{RF}_{\max }(>3 \%)$. This distribution of taxa was characterized by a wide diversity of nectariferous taxa in variable proportions associated with several only-polleniferous species. Among these taxa, two main only-polleniferous taxa, Quercus sp. T1 (Qeurcus sp. (deciduous), Q. ilex and Q. suber) and Cistus sp. T2 (C. creticus, C. monspeliensis and C. salviifolius), were present in all the samples analyzed, followed by Castanea sativa T3 and Fraxinus ornus T4 (PR > 90\%). Additionally, 
we did not find a common predominant nectariferous taxon, unlike two previous studies [24,29]: "chestnut grove" honey predominated by $C$. sativa with $\mathrm{PR}=100 \%, \mathrm{FR}_{\max }>80 \%$ and $\mathrm{FR}_{\text {mean }}=92.99 \%$ and "spring maquis" honey predominated by the "normal" pollen type of Erica arborea with $\mathrm{PR}=100 \%, \mathrm{FR}_{\max }>45 \%$ and $\mathrm{FR}_{\text {mean }}=47.7 \%$. Quite the contrary, this directly demonstrates a diversity of nectariferous taxa with various pollen representation types: for example, "over-represented" (T7 and T13), "normal" (T5, T6, T8, T9 and T14) and "under-represented" (T17, T18 and T22) pollen types [5].

Table 1. Statistical analysis and biogeographical characteristics of Corsican "spring" honeys' taxa.

\begin{tabular}{|c|c|c|c|c|c|c|c|c|c|}
\hline \multirow{2}{*}{ No ${ }^{a}$} & \multirow{2}{*}{ Type $^{\text {b }}$} & \multirow{2}{*}{ Taxa } & \multirow{2}{*}{$\mathbf{P R}^{\mathrm{c}}$} & \multicolumn{5}{|c|}{ Relative frequency (RF) ${ }^{d}$} & \multirow{2}{*}{$\mathbf{B C}^{\mathrm{g}}$} \\
\hline & & & & Mean & Min. & Max. & SD $^{\mathrm{e}}$ & $\mathrm{CV}^{\mathrm{f}}$ & \\
\hline T1 & $\mathrm{P}$ & Quercus sp. & 100 & 13.2 & 0.8 & 35.7 & 9.7 & 73.8 & $21-35-55-58$ \\
\hline $\mathbf{T 2}$ & $\mathrm{P}$ & Cistus sp. & 100 & 8.5 & 0.3 & 33.3 & 6.3 & 74.4 & $21-29$ \\
\hline T3 & $\mathrm{P}$ & Castanea sativa $^{\mathrm{h}}$ & 90 & 10.3 & 0.3 & 33.8 & 8.7 & 84.2 & 59 \\
\hline T4 & $\mathrm{P}$ & Fraxinus ornus & 90 & 3.3 & 0.3 & 22.3 & 5.0 & 153.2 & 58 \\
\hline T5 & $\mathrm{N}, \mathrm{P}$ & Erica arborea & 85 & 7.8 & 0.2 & 35.5 & 8.7 & 112.3 & 21 \\
\hline T6 & $\mathrm{N}, \mathrm{P}$ & Genista form ${ }^{\mathrm{i}}$ & 83 & 6.0 & 0.3 & 31.5 & 8.0 & 134.8 & $14-21-29-51-62$ \\
\hline T7 & $\mathrm{N}, \mathrm{P}$ & Lotus sp. & 76 & 5.3 & 0.3 & 52.8 & 9.5 & 178.3 & $21-51$ \\
\hline T8 & $\mathrm{N}, \mathrm{P}$ & Salix sp. & 73 & 6.3 & 0.2 & 29.9 & 7.4 & 117.1 & $51-52$ \\
\hline T9 & $\mathrm{N}, \mathrm{P}$ & Trifolium sp. & 71 & 14.2 & 0.4 & 53.5 & 16.8 & 117.9 & $21-31-51$ \\
\hline T10 & $\mathrm{N}, \mathrm{P}$ & Rubus sp. & 71 & 3.6 & 0.4 & 11.7 & 3.4 & 94.6 & $31-35$ \\
\hline T11 & $\mathrm{N}, \mathrm{P}$ & Prunus form ${ }^{\mathrm{j}}$ & 66 & 3.0 & 0.2 & 24.1 & 4.7 & 155.6 & $99-54$ \\
\hline T12 & $\mathrm{P}$ & Eucalyptus sp. & 63 & 2.1 & 0.3 & 15.5 & 3.1 & 148.4 & 99 \\
\hline T13 & $\mathrm{N}, \mathrm{P}$ & Echium sp. & 59 & 10.5 & 0.6 & 71.1 & 15.6 & 148.2 & 31 \\
\hline T14 & $\mathrm{N}, \mathrm{P}$ & Apiaceae & 59 & 4.0 & 0.2 & 17.5 & 4.4 & 109.6 & nd \\
\hline T15 & $\mathrm{P}$ & Actinidia sinensis & 49 & 4.2 & 0.3 & 16.1 & 4.5 & 107.7 & 99 \\
\hline T16 & $\mathrm{N}, \mathrm{P}$ & Brassicaceae others & 49 & 2.8 & 0.3 & 14.7 & 3.3 & 118.6 & nd \\
\hline T17 & $\mathrm{N}, \mathrm{P}$ & Lavandula stoechas & 49 & 1.8 & 0.4 & 10.1 & 2.2 & 124.1 & 21 \\
\hline T18 & $\mathrm{N}, \mathrm{P}$ & Citrus sp. & 44 & 6.1 & 0.2 & 16.1 & 5.2 & 86.6 & 99 \\
\hline T19 & $\mathrm{N}, \mathrm{P}$ & Vicia form & 44 & 3.0 & 0.3 & 11.8 & 3.2 & 107.1 & nd \\
\hline $\mathbf{T 2 0}$ & $\mathrm{P}$ & Pistacia lentiscus & 44 & 3.0 & 0.5 & 9.3 & 2.6 & 88.0 & 29 \\
\hline $\mathbf{T} 21$ & $\mathrm{~N}, \mathrm{P}$ & Asteraceae Galactites form & 44 & 1.9 & 0.2 & 5.2 & 1.7 & 93.6 & 21 \\
\hline T22 & $\mathrm{N}, \mathrm{P}$ & Asphodelus ramosus subsp. ramosus & 44 & 0.7 & 0.2 & 2.9 & 0.7 & 96.5 & 21 \\
\hline $\mathbf{T} 23$ & $\mathrm{P}$ & Scrophulariaceae others & 39 & 0.9 & 0.3 & 4.5 & 1.0 & 114.4 & nd \\
\hline $\mathbf{T 2 4}$ & $\mathrm{P}$ & Phillyrea sp. & 37 & 3.0 & 0.3 & 13.3 & 3.8 & 125.5 & 25 \\
\hline $\mathbf{T 2 5}$ & $\mathrm{P}$ & Olea sp. & 37 & 1.0 & 0.4 & 3.6 & 0.8 & 74.3 & 21 \\
\hline $\mathbf{T 2 6}$ & $\mathrm{N}, \mathrm{P}$ & Viburnum tinus & 34 & 1.9 & 0.3 & 16.2 & 4.2 & 225.9 & 21 \\
\hline $\mathbf{T} 27$ & $\mathrm{~N}, \mathrm{P}$ & Asteraceae (fenestrated type) & 29 & 1.1 & 0.3 & 3.2 & 1.0 & 94.0 & $21-94$ \\
\hline $\mathbf{T 2 8}$ & $\mathrm{N}, \mathrm{P}$ & Rosa sp. & 27 & 1.2 & 0.3 & 4.5 & 1.3 & 108.1 & $31-51$ \\
\hline T29 & $\mathrm{P}$ & Myrtus communis & 24 & 0.9 & 0.3 & 1.6 & 0.5 & 52.0 & 21 \\
\hline T30 & $\mathrm{N}, \mathrm{P}$ & Fabaceae others/Dorycnopis form & 24 & 0.6 & 0.3 & 1.4 & 0.3 & 54.5 & nd \\
\hline T31 & $\mathrm{P}$ & Plantago sp. & 24 & 0.5 & 0.3 & 0.9 & 0.2 & 33.8 & nd \\
\hline T32 & $\mathrm{N}, \mathrm{P}$ & Asteraceae Achillea form & 22 & 0.8 & 0.2 & 2.6 & 0.7 & 94.0 & $21-94$ \\
\hline $\mathbf{T 3 3}$ & $\mathrm{P}$ & Poaceae & 22 & 0.6 & 0.2 & 1.2 & 0.3 & 54.0 & nd \\
\hline T34 & $\mathrm{N}, \mathrm{P}$ & Crataegus monogyna & 20 & 2.1 & 0.3 & 7.9 & 2.7 & 130.8 & 51 \\
\hline
\end{tabular}


Table 1. Cont.

\begin{tabular}{|c|c|c|c|c|c|c|c|c|c|}
\hline \multirow{2}{*}{ No ${ }^{a}$} & \multirow{2}{*}{ Type $^{\text {b }}$} & \multirow{2}{*}{ Taxa } & \multirow{2}{*}{$\mathbf{P R}^{\mathrm{c}}$} & \multicolumn{5}{|c|}{${\text { Relative frequency }(\mathrm{RF})^{\mathrm{d}}}^{\mathrm{d}}$} & \multirow{2}{*}{$\mathrm{BC}^{\mathrm{g}}$} \\
\hline & & & & Mean & Min. & Max. & SD $^{\mathrm{e}}$ & $\mathrm{CV}^{\mathrm{f}}$ & \\
\hline T35 & $\mathrm{N}, \mathrm{P}$ & Jasione montana & 17 & 2.0 & 0.3 & 10.0 & 3.5 & 176.0 & 54 \\
\hline T36 & $\mathrm{N}, \mathrm{P}$ & Rosaceae others & 17 & 1.2 & 0.3 & 3.0 & 1.0 & 85.6 & nd \\
\hline T37 & $\mathrm{N}, \mathrm{P}$ & Asteraceae Dittrichia form & 17 & 1.0 & 0.2 & 1.9 & 0.8 & 74.4 & 21-94 \\
\hline T38 & $\mathrm{N}, \mathrm{P}$ & Rhamnus sp. & 15 & 1.0 & 0.3 & 3.3 & 1.1 & 117.6 & 21 \\
\hline T39 & $\mathrm{N}, \mathrm{P}$ & Psoralea bituminosa & 15 & 0.7 & 0.3 & 1.6 & 0.5 & 75.6 & 31 \\
\hline T40 & $\mathrm{N}, \mathrm{P}$ & Knautia sp. & 15 & 0.5 & 0.3 & 0.9 & 0.3 & 52.8 & 31 \\
\hline T41 & $\mathrm{N}, \mathrm{P}$ & Lupinus angustifolius & 12 & 4.8 & 0.3 & 18.9 & 8.0 & 166.1 & 21 \\
\hline T42 & $\mathrm{P}$ & Cytinus hypocistis & 12 & 0.9 & 0.4 & 1.8 & 0.7 & 77.6 & 29 \\
\hline $\mathbf{T 4 3}$ & $\mathrm{N}, \mathrm{P}$ & Hedera helix & 12 & 0.8 & 0.3 & 1.3 & 0.4 & 46.1 & 65 \\
\hline T44 & $\mathrm{N}, \mathrm{P}$ & Liliaceae others & 12 & 0.4 & 0.3 & 0.6 & 0.2 & 45.8 & nd \\
\hline T45 & $\mathrm{N}, \mathrm{P}$ & Allium sp. & 12 & 0.4 & 0.3 & 0.6 & 0.2 & 42.9 & $21-25$ \\
\hline T46 & $\mathrm{N}, \mathrm{P}$ & Acacia dealbata & 12 & 0.4 & 0.3 & 0.4 & 0 & 13.3 & 99 \\
\hline T47 & $\mathrm{P}$ & Alnus sp. & 7 & 2.3 & 0.3 & 6.1 & 3.3 & 146.5 & 51 \\
\hline T48 & $\mathrm{N}, \mathrm{P}$ & Dorycnium sp. & 10 & 1.5 & 0.3 & 3.2 & 1.2 & 83.3 & 35 \\
\hline T49 & $\mathrm{N}, \mathrm{P}$ & Rosmarinus officinalis & 10 & 1.7 & 0.4 & 3.0 & 1.3 & 77.9 & 21 \\
\hline T50 & $\mathrm{P}$ & Vitis vinifera & 7 & 1.5 & 0.4 & 3.0 & 1.3 & 89.4 & 99 \\
\hline
\end{tabular}

${ }^{a}$ Order of taxa were classified by decreasing presence ratio (PR). ${ }^{b}$ Type of taxa: P, polleniferous taxa; N, nectariferous taxa [5]. ${ }^{\mathrm{c}}$ PR: presence ratio, number of honey samples presented $/ 41$ samples, expressed as \%. ${ }^{\mathrm{d}}$ Mean, Min., Max. values expressed as relative frequency RF (number of specify pollen counted/total pollen counted). ${ }^{\mathrm{e}} \mathrm{SD}$ : standard deviation. ${ }^{\mathrm{f}} \mathrm{CV}$ : coefficient variation. ${ }^{\mathrm{g}}$ Biogeographical Code, according to Battesti [5]: 1-Endemic: 14 Mediterraneo-montane origin; 2-Steno-Mediterranean: 21 Wider stenomedit., 25 Western stenomedit., 29 Western macaronesian stenomedit.; 3-Eury-Mediterranean: 31 Wider eurymedit., 35 Western eurymedit.; 5-Eurasian: 51 Wider eurasian, 52 Eurasian, 54 European-caucasian, 55 European, 58 South east european, 59 Southern European; 6-Atlantic: 62 Subatlantic, 65 Atlantic Mediterranean; 94 sub-Cosmopolitan; 99 Cultivated plants; nd: not defined. ${ }^{\mathrm{h}}$ Castanea sativa, taxa of "over-represented" type, could be considered as only-polleniferous taxon according to its RF $(<40 \%)$ and lower pollen density taking into account its over-represented pollen type [6,24]. ${ }^{\mathrm{i}}$ Genista form contained essentially Genista corsica, and also Cytisus villosus, Calicotome spinosa and Calicotome villosa. ${ }^{\mathrm{j}}$ Prunus form contained Prunus sp. and other fruit tree. * Forty two other determined taxa (PR < 10\%): Populus sp., Boraginaceae others, Rumex sp., Ostrya carpinifolia, Ilex aquifolium, Platanus sp., Silene gallica, Stachys glutinosa, Anthyllis hermanniae, Papaver sp., Urticaceae, Reseda sp., Aesculus hippocastanum, Carpobrotus sp., Cercis siliquastrum, Potentilla form, Ranunculaceae, Corylus avellana, Asteraceae Helichrysum form, Arbutus unedo, Erica others, Cupressaceae, Sambucus ebulus, Anemone hortensis, Smilax aspera, Cynoglossum creticum form, Amaryllidaceae, Cyperaceae, Helleborus lividus subsp. corsicus, Mercurialis annua, Robinia pseudoacacia, Clematis sp., Chenopodiaceae, Caryophyllaceae others, Borago officinalis, Centaurea sp., Verbascum sp., Teucrium sp., Centaurium erythrae, Veronica sp., Asteraceae others, Buxus sempervirens (according to decreasing PR).

According to these considerations, two groups of honeys could therefore be distinguished, based not by their FR distributions, but by characteristic associations of taxa (Table 2, Table S1-supplementary materials). The first group included 18 samples (group I: 1-18) and was characterized by the association of cultivated taxa: Citrus sp. T18 and A. sinensis T15 (PR 100\% in 18 samples) followed by Prunus form T11 and Olea sp. T25. Citrus sp. contained essentially $C$. sinensis $\times$ reticulata, which possessed an under-represented pollen type, principally due to nectar secretion of Citrus sp. flowers, often before the maturity of stamens. Citrus pollen varied between $0.2 \%$ and $16.1 \%$, with an average 
of $6.1 \%$. The second group (group II: 23 samples, 19-41) was characterized by the absence of a Citrus sp./A. sinensis association and the significant presence of $A$. ramosus $\mathbf{T} 22$ associated with Pistacia lentiscus T20, Phillyrea sp. T24, Apiaceae T14 and Brassicaceae T16. A. ramosus displayed an extreme "under-represented" pollen type due to the flower form (nectar protected by a large base of long stamens that prevented contact with pollen during bee foraging) and the large pollen size. Asphodelus pollen was present in two samples of group I $(0.5 \%-1.3 \%)$ and 16 samples of group II $(0.2 \%-2.9 \%)$.

In the case of honey with the "under-represented" pollen type, the contribution of other nectariferous species could not be discounted. It had to note that some honeys samples possessed dominant nectariferous taxa (RF $>45 \%)$ : Trifolium sp. T9 for sample 2 and 3, Echium sp. T13 for sample 4 and Lotus sp. T7 for sample 38. The nectar contribution of these taxa could not be neglected. Otherwise, several taxa might take part in the honey composition for their high RF in the pollen spectrum: Trifolium sp. T9 and E. arborea $\mathbf{T} 5$ were characteristic for both groups $\left(\mathrm{RF}_{\max } 53.5 \%\right.$ and $35.5 \%$ for group I and $44.1 \%$ and $29.9 \%$ for group II, respectively); Echium sp. T13, Prunus form T11 and Viburnum tinus $\mathbf{T 2 6}$ possessed a higher $\mathrm{RF}_{\max }$ in group I (71.1\%, 24.1\% and 16.2\%, respectively) than in group II $(30.1 \%, 3.3 \%$ and 3.7\%, respectively), while Lotus sp. T7, Genista form T6, Salix sp. T8, Lupinus angustifolius T41 and Apiaceae T14 were higher in group II (FR $\mathrm{F}_{\max }: 52.8 \%$,

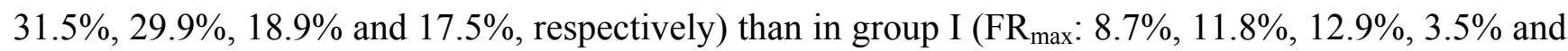
$4.5 \%$, respectively).

A quantitative analysis showed that 32 samples possessed a pollen density between 20 and $100 \times 10^{3} \mathrm{PG} / 10 \mathrm{~g}$, eight samples were between 100 and $300 \times 10^{3} \mathrm{PG} / 10 \mathrm{~g}$ and one sample (23) could be distinguished by high pollen density $\left(600 \times 10^{3} \mathrm{PG} / 10 \mathrm{~g}\right)$. Compared with the previous studies of Corsican "chestnut grove" and "Erica arborea spring maquis" honey $\left(636.6 \times 10^{3} \mathrm{PG} / 10 \mathrm{~g}\right.$ and $177 \times 10^{3} \mathrm{PG} / 10 \mathrm{~g}$, respectively), the "spring" honey displayed a lower pollen density $\left(90 \times 10^{3} \mathrm{PG} / 10 \mathrm{~g}\right)[24,29]$. Excluding sample 23, the average pollen density of "clementine" honeys $\left(68 \times 10^{3} \mathrm{PG} / 10 \mathrm{~g}\right)$ was slightly lower than that of other Corsican "spring" honeys $\left(84 \times 10^{3} \mathrm{PG} / 10 \mathrm{~g}\right)$ (Table 2). The decreasing pollen richness was in accordance with the pollen representation type in the spectrum of the predominant nectariferous taxa: "over-represented" (C. sativa), "normal" (E. arborea) and "under-represented" (Citrus sp.) types. 
Table 2. Melissopalynological and physico-chemical characteristics of Corsican "clementine" honeys and other "spring" honeys.

\begin{tabular}{|c|c|c|c|c|c|c|c|c|c|c|c|c|c|c|}
\hline \multirow[b]{3}{*}{ No. ${ }^{\text {a }}$} & \multirow[b]{3}{*}{ Type $^{\text {b }}$} & \multirow[t]{3}{*}{ ssopalynological Data } & \multicolumn{6}{|c|}{$\begin{array}{c}\text { Group I-“Clementine" Honeys } \\
18 \text { Samples (1-18) }\end{array}$} & \multicolumn{6}{|c|}{$\begin{array}{c}\text { Group II-Other "Spring" Honeys } \\
23 \text { Samples (19-41) }\end{array}$} \\
\hline & & & \multicolumn{6}{|c|}{$\mathbf{R F}^{\mathbf{c}}$} & \multicolumn{6}{|c|}{$\mathbf{R F}^{\mathrm{c}}$} \\
\hline & & & PR & Mean & Min. & Max. & SD & $\mathbf{C V}$ & PR & Mean & Min. & Max. & SD & CV \\
\hline \multicolumn{15}{|c|}{ Main nectariferous taxa } \\
\hline $\mathrm{T} 18$ & $\mathrm{~N}, \mathrm{P}$ & Citrus sp. & 100 & 6.1 & 0.2 & 16.1 & 5.2 & 86.6 & - & - & - & - & - & - \\
\hline $\mathrm{T} 5$ & $\mathrm{~N}, \mathrm{P}$ & Erica arborea & 89 & 7.5 & 0.3 & 35.5 & 10.2 & 135.4 & 83 & 8.0 & 0.2 & 29.7 & 7.6 & 94.9 \\
\hline $\mathrm{T} 8$ & $\mathrm{~N}, \mathrm{P}$ & Salix sp. & 78 & 5.8 & 0.6 & 12.9 & 4.5 & 77.7 & 70 & 6.7 & 0.2 & 29.9 & 9.3 & 139.4 \\
\hline $\mathrm{T} 11$ & $\mathrm{~N}, \mathrm{P}$ & Prunus form & 78 & 4.8 & 0.2 & 24.1 & 6.1 & 128.4 & 57 & 1.2 & 0.3 & 3.3 & 0.9 & 75.5 \\
\hline $\mathrm{T} 7$ & $\mathrm{~N}, \mathrm{P}$ & Lotus sp. & 72 & 2.7 & 0.4 & 8.7 & 2.5 & 91.9 & 78 & 7.2 & 0.3 & 52.8 & 12.1 & 167.5 \\
\hline $\mathrm{T} 10$ & $\mathrm{~N}, \mathrm{P}$ & Rubus sp. & 67 & 2.3 & 0.4 & 6.5 & 1.8 & 77.9 & 74 & 4.5 & 0.4 & 11.7 & 4.0 & 88.4 \\
\hline T9 & $\mathrm{N}, \mathrm{P}$ & Trifolium sp. & 67 & 12.9 & 0.5 & 53.5 & 19.4 & 150.7 & 74 & 15.2 & 0.4 & 44.1 & 15.2 & 100.2 \\
\hline T6 & $\mathrm{N}, \mathrm{P}$ & Genista form & 67 & 3.1 & 0.3 & 11.8 & 3.4 & 107.1 & 96 & 7.5 & 0.6 & 31.5 & 9.4 & 125.3 \\
\hline $\mathrm{T} 13$ & $\mathrm{~N}, \mathrm{P}$ & Echium sp. & 44 & 19.3 & 1.3 & 71.1 & 23.3 & 121.0 & 70 & 6.1 & 0.6 & 30.1 & 7.5 & 123.2 \\
\hline $\mathrm{T} 26$ & $\mathrm{~N}, \mathrm{P}$ & Viburnum tinus & 22 & 4.5 & 0.3 & 16.2 & 7.8 & 174.5 & 43 & 0.8 & 0.3 & 3.7 & 1.1 & 127.7 \\
\hline $\mathrm{T} 14$ & $\mathrm{~N}, \mathrm{P}$ & Apiaceae & 28 & 2.2 & 0.2 & 4.5 & 2.0 & 88.8 & 83 & 4.5 & 0.3 & 17.5 & 4.8 & 106.0 \\
\hline $\mathrm{T} 22$ & $\mathrm{~N}, \mathrm{P}$ & Asphodelus ramosus subsp. ramosus & 11 & 0.9 & 0.5 & 1.3 & 0.5 & 57.3 & 70 & 0.7 & 0.2 & 2.9 & 0.7 & 104.1 \\
\hline $\mathrm{T} 16$ & $\mathrm{~N}, \mathrm{P}$ & Brassicaceae others & 33 & 1.2 & 0.3 & 2.7 & 1.0 & 77.9 & 61 & 3.5 & 0.4 & 14.7 & 3.8 & 108.2 \\
\hline $\mathrm{T} 17$ & $\mathrm{~N}, \mathrm{P}$ & Lavandula stoechas & 39 & 1.7 & 0.5 & 4.4 & 1.4 & 81.9 & 57 & 1.8 & 0.4 & 10.1 & 2.6 & 142.4 \\
\hline T19 & $\mathrm{N}, \mathrm{P}$ & Vicia form & 28 & 2.3 & 0.7 & 6.0 & 2.3 & 102.4 & 57 & 3.3 & 0.3 & 11.8 & 3.6 & 107.6 \\
\hline \multirow[t]{2}{*}{$\mathrm{T} 41$} & $\mathrm{~N}, \mathrm{P}$ & Lupinus angustifolius & 17 & 1.5 & 0.3 & 3.5 & 1.8 & 119.1 & 9 & 9.8 & 0.7 & 18.9 & 12.9 & 131.6 \\
\hline & & Other nectariferous taxa & 100 & 3.3 & 0.3 & 11.0 & 3.0 & 89.9 & 100 & 5.2 & 1.0 & 10.0 & 2.8 & 53.5 \\
\hline \multicolumn{15}{|c|}{ Main only-polleniferous taxa } \\
\hline $\mathrm{T} 15$ & $\mathrm{P}$ & Actinidia sinensis & 100 & 4.6 & 0.3 & 16.1 & 4.6 & 99.7 & 9 & 0.5 & 0.3 & 0.7 & 0.3 & 55.3 \\
\hline $\mathrm{T} 2$ & $\mathrm{P}$ & Cistus sp. & 100 & 6.9 & 0.3 & 20.4 & 5.2 & 76.0 & 100 & 9.8 & 0.3 & 33.3 & 6.9 & 70.9 \\
\hline $\mathrm{T} 1$ & $\mathrm{P}$ & Quercus sp. & 100 & 16.5 & 0.8 & 35.7 & 10.7 & 64.9 & 100 & 10.6 & 1.3 & 29.0 & 8.1 & 77.2 \\
\hline $\mathrm{T} 3$ & $\mathrm{P}$ & Castanea sativa & 89 & 7.9 & 0.3 & 25.0 & 7.3 & 91.7 & 91 & 12.1 & 0.3 & 33.8 & 9.4 & 77.4 \\
\hline $\mathrm{T} 4$ & $\mathrm{P}$ & Fraxinus ornus & 89 & 6.1 & 0.5 & 22.3 & 6.6 & 108.4 & 91 & 1.1 & 0.3 & 3.3 & 1.0 & 87.6 \\
\hline $\mathrm{T} 12$ & $\mathrm{P}$ & Eucalyptus sp. & 72 & 3.3 & 0.4 & 15.5 & 4.1 & 125.9 & 57 & 1.0 & 0.3 & 3.4 & 1.0 & 99.3 \\
\hline $\mathrm{T} 25$ & $\mathrm{P}$ & Olea sp. & 50 & 0.8 & 0.4 & 1.3 & 0.3 & 38.9 & 26 & 1.3 & 0.6 & 3.6 & 1.1 & 84.3 \\
\hline $\mathrm{T} 20$ & $\mathrm{P}$ & Pistacia lentiscus & 11 & 0.6 & 0.6 & 0.6 & 0.0 & 8.2 & 70 & 3.3 & 0.5 & 9.3 & 2.7 & 80.4 \\
\hline \multirow[t]{3}{*}{$\mathrm{T} 24$} & $\mathrm{P}$ & Phillyrea sp. & 17 & 4.7 & 0.3 & 13.3 & 7.4 & 157.4 & 52 & 2.6 & 0.4 & 9.9 & 2.7 & 103.5 \\
\hline & & Other only-polleniferous taxa & 100 & 1.9 & 0.3 & 8.9 & 2.1 & 110.0 & 78 & 1.7 & 0.3 & 8.5 & 2.0 & 117.8 \\
\hline & & Pollen density $\left(10^{3} \mathrm{PG} / 10 \mathrm{~g}\right)^{\mathrm{d}}$ & & 68 & 20 & 202 & 52 & 77 & & 107 & 22 & 603 & 126 & 118 \\
\hline \multicolumn{15}{|c|}{ Physico-chemical data ${ }^{e}$} \\
\hline & & Color & & 26.4 & 11.0 & 55.0 & 13.6 & 51.5 & & 33.3 & 18.0 & 71.0 & 16.3 & 48.7 \\
\hline & & Electrical conductivity & & 0.25 & 0.15 & 0.42 & 0.07 & 27.72 & & 0.24 & 0.13 & 0.45 & 0.09 & 36.96 \\
\hline
\end{tabular}

${ }^{a}$ Taxa number is given in Table $1 .{ }^{\mathrm{b}}$ Type of taxa: $\mathrm{P}$, polleniferous taxa; $\mathrm{N}$, nectariferous taxa [5]. ${ }^{\mathrm{c}}$ Mean, Min., Max. values expressed as relative frequency RF (number of specify pollen counted/total pollen counted). ${ }^{\mathrm{d}}$ Pollen density expressed as the absolute number of pollen grains in $10 \mathrm{~g}$ of honey $\left(10^{3} \mathrm{PG} / 10 \mathrm{~g}\right) .{ }^{\mathrm{e}}$ Unity of parameters: colour (mm Pfund); electrical conductivity (mS/cm). 


\subsection{Physicochemical Characteristics of Corsican "Spring” Honeys}

Corsican "spring" honeys possessed light to very light colors. The mean value of coloration was $30.0 \pm 15.4 \mathrm{~mm}$ Pfund, with great variation between 11.0 and $71.0 \mathrm{~mm}$ Pfund (Table 2). The two groups exhibited quite similar coloration values: $26.4 \pm 13.6 \mathrm{~mm}$ Pfund for "clementine" honeys and $33.3 \pm 16.3 \mathrm{~mm}$ Pfund for the other "spring" honeys. For each group, nine samples possessed a very light coloration value ( $<20.0 \mathrm{~mm}$ Pfund). Only one sample (17) of "clementine" honeys had a coloration value $>50.0 \mathrm{~mm}$ Pfund while five samples $(23,35,38,39$ and 41) of other "spring" honeys possessed coloration values between 50.0 and $71.0 \mathrm{~mm}$ Pfund.

The average electrical conductivity value of the honey samples was $0.25 \pm 0.08 \mathrm{mS} / \mathrm{cm}$ with a variation of $0.13-0.45 \mathrm{mS} / \mathrm{cm}$ (Table 2). The electrical conductivity of the two groups was also quite similar: $0.25 \pm 0.07 \mathrm{mS} / \mathrm{cm}$ for "clementine" honeys (range: $0.15-0.42 \mathrm{mS} / \mathrm{cm}$ ) and $0.24 \pm 0.09 \mathrm{mS} / \mathrm{cm}$ for other "spring" honeys (range: $0.13-0.45 \mathrm{mS} / \mathrm{cm}$ ). Only three samples (17, 34 and 41 ) of these honeys had medium electrical conductivity $(>0.4 \mathrm{mS} / \mathrm{cm})$.

The coloration and electrical conductivity values of Corsican "spring" honeys were lower than those of "chestnut grove" and "Erica arborea spring maquis" honey ranges [5,24,29].

\subsection{Chemical Variability of Corsican "Spring” Honeys}

GC and GC/MS analysis of the headspaces of Corsican "spring" honeys allowed the identification of 43 compounds that accounted for $71.5 \%-96.8 \%$ of the total volatile composition (Table 3, Table S2-supplementary materials). It should be noted that the volatile fraction of "spring" honeys is rich in aldehyde $(22.1 \%-63.1 \%)$ and alcohol $(2.8 \%-40.2 \%)$ components.

To synthesize the chemical data, PCA was used to examine the relative distribution of the matrix of "spring" honey samples according to their volatile chemical compositions. The analyses included 17 compounds: two hydrocarbons $(\mathbf{C 2}$ and C12), eight aldehydes $(\mathbf{C 5}, \mathbf{C 9}, \mathbf{C 1 4}, \mathbf{C 2 5}, \mathbf{C 2 7}, \mathbf{C 2 8}, \mathbf{C 3 1}$ and C32), two ketones (C23 and C24), two esters (C38 and C41), two oxides (C39 and C42) and one alcohol (C37). As shown in Figure 1a, the principal factorial plane (axes 1 and 2) accounted for $58.91 \%$ of the entire variability of the honey samples. Dimension $1(42.24 \%)$ correlated negatively C39 and negatively with other compounds. Dimension $2(16.67 \%)$ correlated negatively with two hydrocarbons (C2 and C12), two aldehydes (C5 and C14) and one oxide $\mathbf{C 4 2}$ and positively with with two ketones (C23 and C24), three aldehydes (C25, C27 and C28), one ester $\mathbf{C 3 8}$ and one oxide other compounds.

The plot established according to the first two principal components suggested the existence of two main groups (Figure 1b). Group I contained 17 samples (1-17), which corresponded to the group of "clementine" honeys (except sample 18). This group I was characterized by the presence of lilac aldehyde isomers (C25, C27 and C28), p-menth-1-en-9-al isomers (C31 and C32) and methyl anthranilate C38, which were absent in the other honey samples (group II). It was rich in furan compounds (group I: $26.2 \%$ versus group II: $7.6 \%$ ), but not in phenolic components (group I: $29.4 \%$ versus group II: $40.0 \%)$. Aldehyde components were also higher in group I (49.1\%) than in group II (39.7\%). Group II could be divided into subgroups IIa (five samples: 20, 33, 36, 38 and 39) and IIb (18 samples: 18, 19, 21-32, 34, 37, 40 and 41). These two subgroups were characterized by a greater 
abundance of phenolic compounds (group IIa: 39.9\% and group IIb: 43.0\%), but group IIa displayed a higher value for linear compounds (group IIa: 26.2\% and group IIb: 19.2\%). Additionally, subgroup IIa had a higher amount of aldehyde (group IIa: $40.7 \%$ and group IIb: $12.8 \%$ ) and alcohol (group IIa: $35.6 \%$ and group IIb: 9.2\%) compounds than subgroup IIb. This latter group displayed a greater abundance of ketones (group IIa: 4.3\% and group IIb: 22.5\%). Finally, sample 35 was characterized by $32.8 \%$ of hydrocarbons, whereas the abundance of hydrocarbons was not $>25 \%$ in the other honey samples.

Table 3. Chemical composition of volatile fraction of Corsican "spring" honeys.

\begin{tabular}{|c|c|c|c|c|c|c|c|c|c|c|c|c|}
\hline \multirow[b]{3}{*}{ No ${ }^{a}$} & \multirow[b]{3}{*}{ Components } & \multirow[b]{3}{*}{$\mathbf{R I}^{\mathbf{b}}$} & \multicolumn{3}{|c|}{ Group I "Clementine" Honeys ${ }^{c}$} & \multicolumn{6}{|c|}{ Group II "Not-Clementine" Honeys c } & \multirow[b]{3}{*}{ Sample 35} \\
\hline & & & \multirow[b]{2}{*}{ Mean \pm SD $^{d}$} & \multirow[b]{2}{*}{ Min. } & \multirow[b]{2}{*}{ Max. } & \multicolumn{3}{|c|}{ IIa } & \multicolumn{3}{|c|}{ IIb } & \\
\hline & & & & & & Mean \pm SD $^{d}$ & Min. & Max. & Mean \pm SD $^{d}$ & Min. & Max. & \\
\hline C1 & 3-Methyl-3-buten-1-ol & 704 & $2.9 \pm 2.76$ & 0.3 & 11.3 & $1.8 \pm 1.19$ & 0.7 & 3.7 & $1.8 \pm 1.44$ & 0.4 & 6.0 & 2.8 \\
\hline $\mathbf{C 2}$ & Methyl-benzene & 741 & $6.5 \pm 4.45$ & 1.5 & 15.6 & $4.1 \pm 2.08$ & 2.4 & 7.1 & $6.2 \pm 4.08$ & 1.5 & 17.3 & 10.4 \\
\hline $\mathbf{C 3}$ & Hexanal & 773 & $1.1 \pm 0.46$ & 0.5 & 2.0 & $1.6 \pm 2.62$ & 0.1 & 6.3 & $1.6 \pm 1.13$ & 0.3 & 4.5 & 1.9 \\
\hline $\mathrm{C} 4$ & Octane & 790 & $1.4 \pm 1.08$ & 0.3 & 4.7 & $0.9 \pm 0.77$ & 0.3 & 2.2 & $2.6 \pm 1.63$ & 0.7 & 5.6 & 1.4 \\
\hline C5 & 3-Furaldehyde & 800 & $2.8 \pm 1.56$ & 0.6 & 5.9 & $3.5 \pm 1.37$ & 2.5 & 5.9 & $3.5 \pm 1.78$ & 1.9 & 8.3 & 18.5 \\
\hline C6 & 2-Methyl butanoic acid & 858 & $0.8 \pm 0.91$ & 0.1 & 3.3 & $4.6 \pm 3.21$ & 1.1 & 7.6 & $2.9 \pm 4.59$ & 0.1 & 20.4 & 2.4 \\
\hline C7 & 2-Methyl octane & 873 & $0.4 \pm 0.28$ & 0.1 & 0.9 & $0.5 \pm 0.44$ & 0.1 & 1.2 & $0.7 \pm 0.38$ & 0.1 & 1.7 & 1.6 \\
\hline C8 & Nonane & 893 & $0.7 \pm 0.65$ & 0.2 & 2.5 & $1.2 \pm 0.35$ & 0.8 & 1.7 & $1.5 \pm 0.92$ & 0.2 & 3.5 & 3.8 \\
\hline C9 & Benzaldehyde & 924 & $5.5 \pm 3.56$ & 2.4 & 17.9 & $10.4 \pm 3.85$ & 5.4 & 14.8 & $8.8 \pm 4.89$ & 2.5 & 18.4 & 3.0 \\
\hline C10 & Hexanoic acid & 969 & $0.7 \pm 0.25$ & 0.4 & 1.4 & $1.7 \pm 1.68$ & 0.3 & 3.9 & $1.2 \pm 0.75$ & 0.4 & 3.3 & - \\
\hline C11 & Octanal & 982 & $1.0 \pm 0.47$ & 0.2 & 2.1 & $0.6 \pm 0.12$ & 0.5 & 0.7 & $1.6 \pm 1.45$ & 0.5 & 6.6 & 1.0 \\
\hline C12 & 2,2,4,6,6-Pentamethylheptane & 992 & $1.1 \pm 0.60$ & 0.4 & 2.4 & $0.6 \pm 0.53$ & 0.1 & 1.3 & $2.3 \pm 1.83$ & 0.2 & 5.2 & 15.6 \\
\hline $\mathrm{C} 13$ & $p$-Methylanisol & 995 & $0.9 \pm 1.13$ & 0.1 & 4.9 & $1.1 \pm 1.10$ & 0.3 & 3.0 & $0.9 \pm 1.48$ & 0.1 & 6.1 & - \\
\hline C14 & Phenylacetaldehyde & 1006 & $10.1 \pm 10.68$ & 0.8 & 39.1 & $16.5 \pm 6.93$ & 7.2 & 25.7 & $21.2 \pm 7.83$ & 3.7 & 36.2 & 13.0 \\
\hline C15 & $p$-Cymene & 1008 & $0.7 \pm 0.29$ & 0.1 & 1.0 & $0.9 \pm 0.42$ & 0.6 & 1.2 & - & - & - & - \\
\hline C16 & Acetophenone & 1037 & $0.2 \pm 0.08$ & 0.1 & 0.4 & $0.4 \pm 0.21$ & 0.2 & 0.5 & $0.3 \pm 0.10$ & 0.2 & 0.4 & - \\
\hline $\mathrm{C} 17$ & trans-Furanoid-linaloxide & 1049 & $1.5 \pm 1.00$ & 0.8 & 4.0 & $1.3 \pm 1.25$ & 0.5 & 3.5 & $2.4 \pm 1.20$ & 1.0 & 6.3 & - \\
\hline C18 & cis-Furanoid-linaloxide & 1064 & $1.1 \pm 0.40$ & 0.7 & 2.0 & $1.0 \pm 0.29$ & 0.7 & 1.4 & $1.1 \pm 0.26$ & 0.5 & 1.6 & - \\
\hline C19 & $\beta$-Phenylethanol & 1077 & $4.2 \pm 1.54$ & 2.2 & 5.8 & $1.6 \pm 0.00$ & 1.6 & 1.6 & $3.3 \pm 1.67$ & 2.1 & 5.8 & - \\
\hline $\mathrm{C20}$ & Nonanal & 1079 & $2.7 \pm 1.73$ & 0.9 & 7.6 & $1.8 \pm 1.28$ & 0.4 & 3.5 & $3.0 \pm 2.29$ & 0.5 & 7.2 & 2.9 \\
\hline $\mathrm{C} 21$ & Linalol & 1084 & $2.4 \pm 1.82$ & 0.2 & 6.6 & $1.3 \pm 1.62$ & 0.3 & 3.2 & $12.5 \pm 10.42$ & 2.1 & 32.3 & $\operatorname{tr}$ \\
\hline $\mathrm{C} 22$ & Hotrienol & 1085 & $4.1 \pm 4.39$ & 0.7 & 10.5 & - & - & - & $9.7 \pm 0.00$ & 9.7 & 9.7 & - \\
\hline $\mathrm{C} 23$ & Isophorone & 1087 & $2.8 \pm 1.54$ & 0.2 & 4.9 & $18.2 \pm 8.02$ & 8.8 & 29.3 & $3.3 \pm 3.5$ & 0.1 & 9.6 & - \\
\hline $\mathrm{C} 24$ & 4-Oxoisophorone & 1102 & $0.9 \pm 0.33$ & 0.3 & 1.4 & $4.2 \pm 1.87$ & 2.3 & 6.4 & $1.5 \pm 0.99$ & 0.3 & 5.0 & - \\
\hline $\mathrm{C} 25$ & $\left(2 S, 2^{\prime} S, 5^{\prime} S\right)$-Lilac aldehyde & 1112 & $5.4 \pm 2.36$ & 1.3 & 8.9 & - & - & - & $1.5 \pm 0.00$ & 1.5 & 1.5 & - \\
\hline $\mathrm{C26}$ & Dihydrolinalool & 1116 & $1.2 \pm 0.66$ & 0.5 & 3.0 & - & - & - & $1.1 \pm 0.00$ & 1.1 & 1.1 & - \\
\hline $\mathrm{C} 27$ & $\left(2 R, 2^{\prime} S, 5^{\prime} S\right)$-Lilac aldehyde & 1121 & $10.5 \pm 3.66$ & 4.9 & 16.5 & - & - & - & $2.4 \pm 0.00$ & 2.4 & 2.4 & - \\
\hline $\mathrm{C28}$ & $\left(2 R, 2^{\prime} R, 5^{\prime} S\right)$-Lilac aldehyde & 1134 & $4.8 \pm 1.85$ & 2.2 & 8.1 & - & - & - & $1.1 \pm 0.00$ & 1.1 & 1.1 & - \\
\hline $\mathrm{C29}$ & Octanoic acid & 1167 & $1.7 \pm 1.55$ & 0.3 & 6.0 & $0.9 \pm 0.38$ & 0.3 & 1.3 & $1.6 \pm 0.78$ & 0.7 & 3.8 & 4.7 \\
\hline C30 & Decanal & 1174 & $1.2 \pm 0.67$ & 0.2 & 2.8 & $0.6 \pm 0.25$ & 0.2 & 0.8 & $1.5 \pm 0.54$ & 0.6 & 2.3 & - \\
\hline C31 & $p$-Menth-1-en-9-al (isomer 1) & 1184 & $1.9 \pm 0.41$ & 1.2 & 2.7 & - & - & - & - & - & - & - \\
\hline $\mathrm{C} 32$ & $p$-Menth-1-en-9-al (isomer 2) & 1186 & $1.7 \pm 0.46$ & 0.5 & 2.5 & - & - & - & - & - & - & - \\
\hline C33 & $p$-Anisaldehyde & 1208 & $0.7 \pm 1.19$ & 0.1 & 4.6 & $0.9 \pm 0.37$ & 0.3 & 1.1 & $0.4 \pm 0.21$ & 0.2 & 0.8 & - \\
\hline C34 & 2,3,5-Trimethylphenol & 1248 & $0.4 \pm 0.30$ & 0.1 & 1.1 & $1.0 \pm 0.69$ & 0.4 & 2.0 & $0.8 \pm 0.68$ & 0.1 & 2.0 & - \\
\hline
\end{tabular}


Table 3. Cont.

\begin{tabular}{|c|c|c|c|c|c|c|c|c|c|c|c|c|}
\hline \multirow[b]{3}{*}{ No ${ }^{\text {a }}$} & \multirow[b]{3}{*}{ Components } & \multirow[b]{3}{*}{$\mathbf{R I}^{\mathbf{b}}$} & \multicolumn{3}{|c|}{ Group I “Clementine” Honeys c } & \multicolumn{6}{|c|}{ Group II "Not-Clementine" Honeys c } & \multirow[b]{3}{*}{ Sample 35} \\
\hline & & & \multirow[b]{2}{*}{ Mean \pm SD $^{d}$} & \multirow[b]{2}{*}{ Min } & \multirow[b]{2}{*}{ Max } & \multicolumn{3}{|c|}{ IIa } & \multicolumn{3}{|c|}{ IIb } & \\
\hline & & & & & & Mean \pm SD $^{d}$ & Min & $\operatorname{Max}$ & Mean \pm SD $^{d}$ & Min & Max & \\
\hline C35 & 4- $n$-Propylanisol & 1264 & $1.6 \pm 1.78$ & 0.2 & 5.7 & $2.4 \pm 1.29$ & 0.8 & 4.3 & $3.8 \pm 2.45$ & 1.4 & 6.3 & - \\
\hline $\mathrm{C} 36$ & Nonanoic acid & 1271 & $2.7 \pm 1.39$ & 0.5 & 4.9 & $2.6 \pm 0.94$ & 1.4 & 3.7 & $3.1 \pm 1.24$ & 1.3 & 6.4 & - \\
\hline $\mathrm{C} 38$ & Methyl anthranilate & 1300 & $1.4 \pm 0.96$ & 0.2 & 3.5 & - & - & - & - & - & - & - \\
\hline C39 & $\begin{array}{l}\text { cis-p-Mentha-1(7),8-dien-1- } \\
\text { hydroperoxide }\end{array}$ & 1348 & $0.4 \pm 0.14$ & 0.2 & 0.7 & - & - & - & - & - & - & - \\
\hline C40 & Decanoic acid & 1362 & $1.2 \pm 0.45$ & 0.6 & 2.1 & $1.3 \pm 0.75$ & 0.1 & 1.9 & $1.7 \pm 1.50$ & 0.6 & 6.8 & 3.8 \\
\hline C41 & Methyl 3,5-dimethoxybenzoate & 1494 & - & - & - & $0.4 \pm 0.26$ & 0.2 & 0.7 & $0.5 \pm 0.19$ & 0.3 & 0.8 & - \\
\hline \multirow[t]{3}{*}{$\mathbf{C 4 3}$} & Tricosane & 2305 & $0.3 \pm 0.17$ & 0.1 & 0.5 & $0.5 \pm 0.00$ & 0.5 & 0.5 & $0.5 \pm 0.22$ & 0.2 & 0.7 & - \\
\hline & Total identification (\%) & & $84.2 \pm 6.95$ & 71.5 & 94.5 & $91.2 \pm 5.43$ & 84.2 & 96.8 & $86.3 \pm 5.49$ & 78.8 & 96.7 & 86.8 \\
\hline & Total peak area $\left(10^{6}\right)^{e}$ & & $3.8 \pm 1.96$ & 1.3 & 7.4 & $2.9 \pm 1.27$ & 1.6 & 4.5 & $2.4 \pm 1.08$ & 0.8 & 4.4 & 0.3 \\
\hline & Hydrocarbons & & $10.6 \pm 5.23$ & 4.7 & 20.8 & $7.7 \pm 3.59$ & 4.8 & 13.5 & $13.3 \pm 5.88$ & 5.0 & 23.9 & 32.8 \\
\hline & Oxygenated compounds & & $73.6 \pm 7.23$ & 58.2 & 82.8 & $83.6 \pm 4.11$ & 79.2 & 90.3 & $73.7 \pm 7.56$ & 58.1 & 81.7 & 54.0 \\
\hline & Phenolic compounds & & $29.4 \pm 12.3$ & 12.6 & 59.3 & $43.0 \pm 7.39$ & 34.8 & 53.0 & $39.9 \pm 11.22$ & 23.2 & 60.4 & 26.4 \\
\hline & Furan compounds & & $26.2 \pm 7.85$ & 12.2 & 38.6 & $5.8 \pm 2.83$ & 3.9 & 10.8 & $7.5 \pm 1.99$ & 4.3 & 11.0 & 18.5 \\
\hline & Linear compounds & & $21.0 \pm 7.08$ & 11.3 & 36.6 & $19.2 \pm 3.88$ & 14.8 & 23.4 & $26.2 \pm 9.63$ & 11.3 & 53.4 & 41.9 \\
\hline & Terpenic compounds & & $31.0 \pm 9.88$ & 15.4 & 52.2 & $3.5 \pm 2.64$ & 1.7 & 8.1 & $13.6 \pm 13.05$ & 1.5 & 45.8 & 0 \\
\hline & Ketones & & $2.5 \pm 2.14$ & 0 & 6.0 & $22.5 \pm 9.67$ & 11.6 & 35.7 & $4.3 \pm 3.77$ & 0.8 & 11.6 & 0 \\
\hline & Esters & & $1.4 \pm 0.96$ & 0.2 & 3.5 & $0.2 \pm 0.29$ & 0 & 0.7 & $0.3 \pm 0.27$ & 0 & 0.8 & 0 \\
\hline & Alcohols & & $9.7 \pm 6.17$ & 3.3 & 27.8 & $9.2 \pm 3.3$ & 4.9 & 12.7 & $12.8 \pm 10.40$ & 3.0 & 40.2 & 2.8 \\
\hline & Acids & & $6.8 \pm 3.31$ & 0.4 & 15.4 & $9.7 \pm 5.23$ & 3.0 & 15.1 & $10.2 \pm 5.28$ & 5.6 & 27.0 & 10.9 \\
\hline & Oxides & & $5.2 \pm 2.76$ & 2.5 & 12.6 & $6.3 \pm 2.45$ & 3.0 & 9.3 & $5.5 \pm 2.64$ & 3.3 & 14.3 & 0 \\
\hline
\end{tabular}

${ }^{\mathrm{a}}$ Order of elution is given on apolar coloumn (Rtx-1). ${ }^{\mathrm{b}}$ Retention indice on the Rtx-1 apolar column. ${ }^{\mathrm{c}}$ Group number was given in "Chemical variability of Corsican "spring" honeys". ${ }^{d}$ Means \pm SD, Min. and Max. values expressed as percentages. ${ }^{\mathrm{e}}$ Total peak area was expressed in arbitrary units. 
Figure 1. Principal component analysis (PCA) of Corsican "spring" honey volatile data.

(a) PCA distribution of variable Variables factor map (PCA)

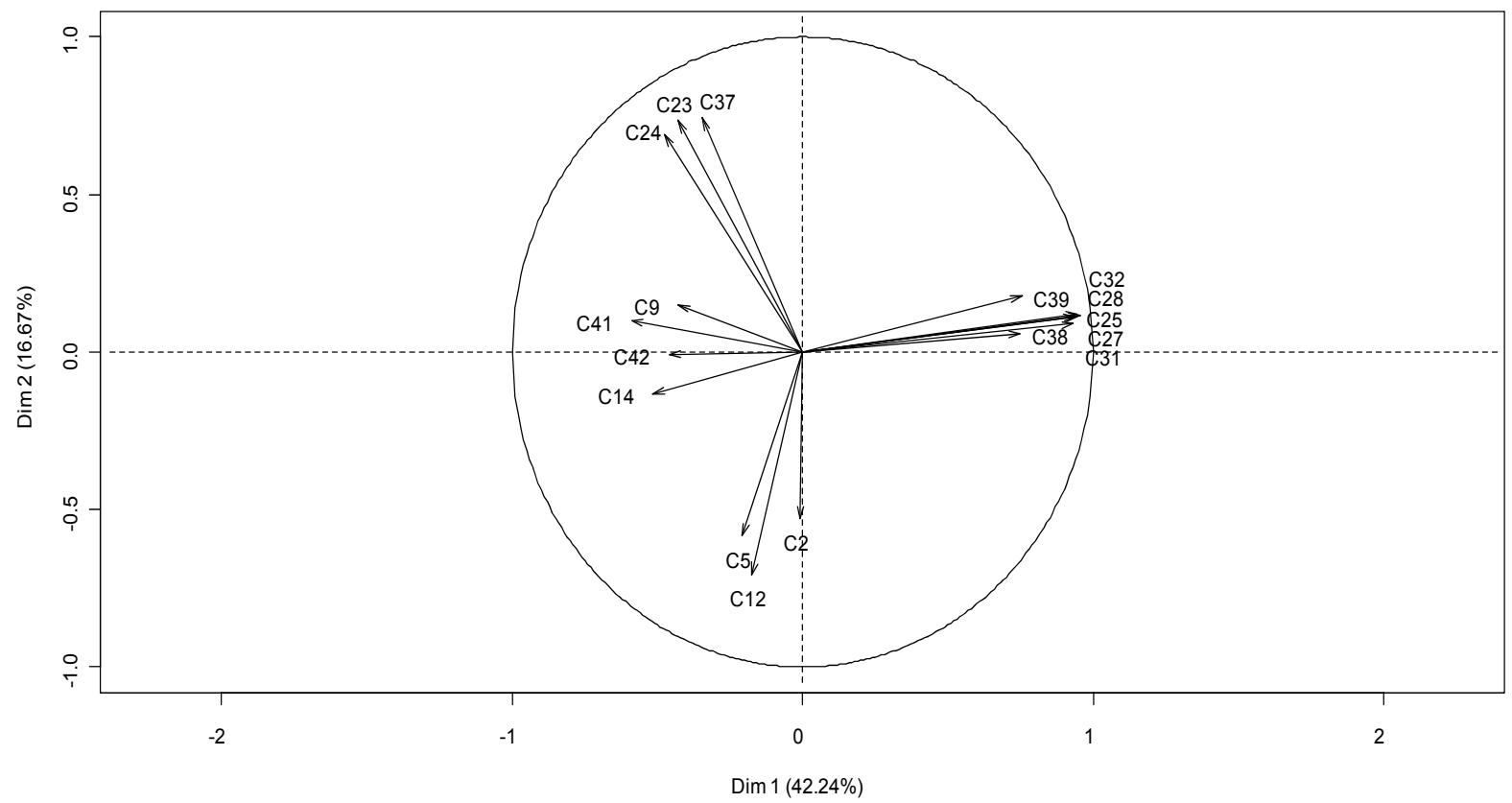

Volatile compounds number corresponding to those of Table 3.

(b) PCA distribution of honey samples (1-41)

Individuals factor map (PCA)

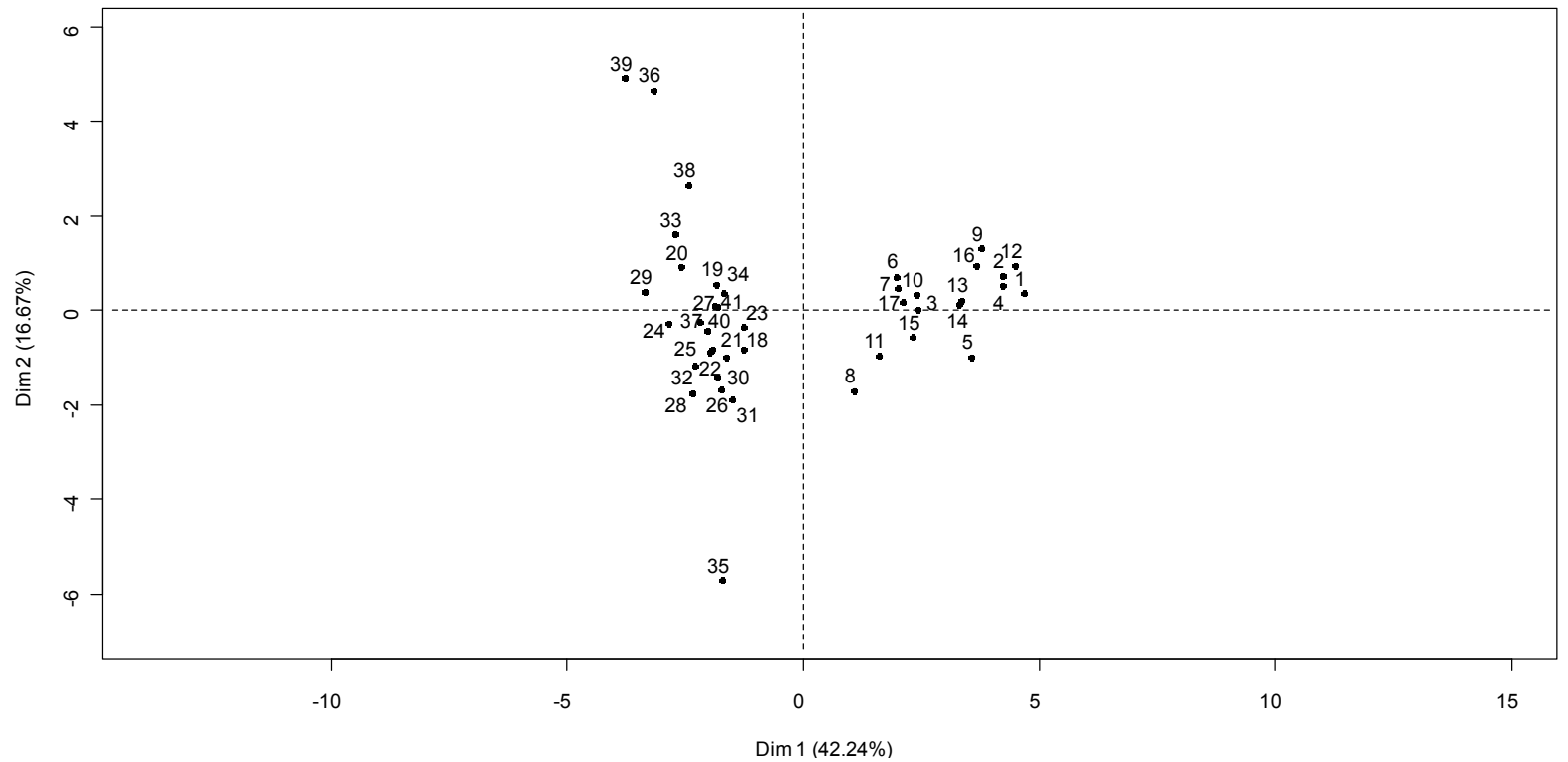




\subsection{Botanical Origin and Volatile Composition of Corsican "Spring” Honeys}

The 17 samples of "clementine" honeys (group I: 1-17) could be distinguished from other "spring" honeys (group II) by the presence of three lilac aldehydes (C25, C27 and C28) and two $p$-menth-1-en-9-al isomers (C31 and C32) (Table 3, Table S2-supplementary materials). These honey samples were dominated by phenolic compounds $(12.6 \%-59.3 \%)$, followed by furan compounds $(12.2 \%-38.6 \%)$ and linear compounds $(11.3 \%-36.6 \%)$. The main components were phenylacetaldehyde C14 (0.8\%-39.1\%), methyl-benzene C2 (1.5\%-15.6\%), (2R,2'S,5'S)-lilac aldehyde C27 (4.9\%-16.5\%), ( $\left.2 S, 2^{\prime} S, 5^{\prime} S\right)$-lilac aldehyde $\mathbf{C 2 5}$ (1.3\%-8.9\%), benzaldehyde C9 $(2.4 \%-17.9 \%)$ and $\left(2 R, 2^{\prime} R, 5^{\prime} S\right)$-lilac aldehyde $\mathbf{C 2 8}(2.2 \%-8.1 \%)$. Low amounts of methyl anthranilate $\mathbf{C 3 8}(0.2 \%-3.5 \%)$ were found in the volatile fraction of the "clementine" honeys analyzed. This component is a known chemical marker of Citrus (species not specified) unifloral honey [13]. Additionally, various linalool derivatives, such as linalool oxides, lilac aldehydes and/or $p$-menth-1-en-9-al isomers, have also been reported as characteristic compounds of citrus unifloral honeys from Spain and Greece [15,16,30-33]. These compounds were also identified in the volatile components of Corsican "clementine" honeys. Conversely, some other linalool derivatives, such as lilac alcohol isomers (previously reported in the Spanish and Greek citrus honeys), were not detected in our honey samples. Alissandrakis et al. [15] showed that methyl anthranilate and lilac aldehydes could be found in honeys of mixed botanical origin with the presence of citrus nectar. These volatile compounds were also detected in the honey samples 2-4 in which were found the $\mathrm{RF}_{\max }$ of Trifolium sp. T9 and Echium sp. T13 taxa. Chemical investigation showed that these honey samples displayed the similar volatile composition of "clementine" honey. As these two taxa could provide great quantity of nectar and pollen [34], it appeared that they played only a polleniferous role in these honey samples.

The volatile composition of $C$. sinensis $\times$ reticulata flowers has not been reported previously. The HS-SPME fraction of clementine flowers is characterized by 29 compounds, which accounted for $75.5 \%-87.0 \%$ of the volatile composition (Table 4$)$. Linalool $(9.6 \%-22.6 \%)$, sabinene $(13.4 \%-19.6 \%)$, dihydrolinalool $(8.5 \%-14.8 \%)$ and myrcene $(5.6 \%-6.5 \%)$ were identified as the main compounds. Linalool and dihydrolinalool were also found in low concentrations in the volatile fraction from "spring clementine" honey samples. Methyl anthranilate was detected in the volatile fraction of Corsican clementine flowers $(0.1 \%-0.3 \%)$ and corresponding honeys.

The decrease in linalool amount and the occurrence of other linalool derivates (hotrienol, linalool oxides, lilac aldehyde isomers and $p$-menth-1-en-9-al isomers) in honey samples could be explained by the enzymatic degradation of linalool by some pathways [15]: (1) linalool can be transformed to 8-hydroxylinalool isomers by enzymatic hydroxylation at the $\mathrm{C} 8$ position, and then hotrienol; (2) 8-hydroxylinalool can be transformed to lilac aldehyde via (E)-8-oxolinalool and lilac alcohols, or p-menth-1-en-9-al via 8-hydroxygeraniol and (3) linalool can also be transformed via 6,7-hydroxylinalool into furanoid linalool oxide isomers under acidic conditions or by heating. These results were in accordance with those previously reported on the volatile fraction of citrus flowers and corresponding honeys [16]. It demonstrated that the flowers from Citrus species (orange, tangerine and sour orange) had high amounts of linalool $(51.6 \%-80.6 \%)$ and that the honeys consisted of more than $80 \%$ of linalool derivatives (lilac aldehydes and lilac alcohols). 
Table 4. Chemical composition of volatile fraction of clementine and asphodel flowers.

\begin{tabular}{|c|c|c|c|c|c|c|c|c|c|}
\hline \multirow{2}{*}{ Components $^{\mathrm{a}}$} & \multirow{2}{*}{$\mathbf{R I}(\text { Lit })^{b}$} & \multirow{2}{*}{$\mathbf{R I}^{\mathrm{c}}$} & \multicolumn{3}{|c|}{ Clementine Flower $^{d}$} & \multicolumn{3}{|c|}{ Asphodel Flower $^{\text {e }}$} & \multirow{2}{*}{ Identification $\mathrm{g}$} \\
\hline & & & $\operatorname{Mean} \pm \mathbf{S D}^{\mathrm{f}}$ & Min. & Max. & $\operatorname{Mean} \pm$ SD $^{\mathrm{f}}$ & Min. & Max. & \\
\hline 3-Furaldehyde & 799 & 800 & - & - & - & $1.0 \pm 0.87$ & 0.5 & 2.7 & RI, MS \\
\hline Furfural & 831 & 836 & - & - & - & $3.5 \pm 1.26$ & 1.7 & 5.2 & RI, MS \\
\hline 2-Furanmethanol & 839 & 842 & - & - & - & $2.1 \pm 1.48$ & 0.8 & 4.7 & RI, MS, Ref \\
\hline Heptanal & 882 & 876 & - & - & - & $5.4 \pm 2.46$ & 3.1 & 9.5 & RI, MS \\
\hline$\alpha$-Thujene & 924 & 922 & $1.2 \pm 0.21$ & 1.0 & 1.4 & - & - & - & RI, MS \\
\hline$\alpha$-Pinene & 932 & 931 & $3.6 \pm 2.46$ & 2.0 & 6.4 & - & - & - & RI, MS \\
\hline Benzaldehyde & 929 & 933 & - & - & - & $2.7 \pm 0.78$ & 1.4 & 3.7 & RI, MS \\
\hline Tetrahydro-citronellene & 937 & 935 & $6.8 \pm 4.90$ & 3.3 & 12.4 & - & - & - & RI, MS, Ref \\
\hline$\beta$-Citronellene & 943 & 940 & $2.2 \pm 0.15$ & 2.0 & 2.3 & - & - & - & RI, MS \\
\hline Octen-3-ol & 962 & 955 & - & - & - & $0.2 \pm 0.05$ & 0.1 & 0.2 & RI, MS \\
\hline Furfuryl acetate & 964 & 959 & - & - & - & $0.7 \pm 0.31$ & 0.5 & 1.3 & RI, MS, Ref \\
\hline Sabinene & 973 & 958 & $16.8 \pm 3.14$ & 13.4 & 19.6 & - & - & - & RI, MS \\
\hline 2-Pentylfuran & 973 & 966 & - & - & - & $0.8 \pm 1.00$ & 0.2 & 2.8 & RI, MS \\
\hline$\beta$-Pinene & 978 & 972 & $1.5 \pm 1.36$ & 0.4 & 3.0 & - & - & - & RI, MS \\
\hline Myrcene & 987 & 979 & $6.1 \pm 0.45$ & 5.6 & 6.5 & - & - & - & RI, MS \\
\hline Octanal & 981 & 980 & - & - & - & $7.0 \pm 3.12$ & 3.5 & 12.6 & RI, MS \\
\hline (Z)-3-Hexenyl acetate & 989 & 984 & - & - & - & $21.6 \pm 14.27$ & 5.2 & 41.8 & RI, MS \\
\hline (E)-3-Hexenyl acetate & 1002 & 994 & - & - & - & $0.8 \pm 0.54$ & 0.1 & 1.5 & RI, MS \\
\hline$\alpha$-Phellandrene & 1002 & 995 & $1.5 \pm 0.23$ & 1.4 & 1.8 & $0.3 \pm 0.12$ & 0.1 & 0.4 & RI, MS \\
\hline$\alpha$-Terpinene & 1013 & 1008 & $0.6 \pm 0.44$ & 0.3 & 1.1 & - & - & - & RI, MS \\
\hline Phenylacetaldehyde & 1012 & 1009 & - & - & - & $0.9 \pm 0.67$ & 0.2 & 2.1 & RI, MS \\
\hline$p$-Cymene & 1015 & 1011 & $0.6 \pm 0.10$ & 0.5 & 0.7 & - & - & - & RI, MS \\
\hline$p$-Menth-1-ene & 1017 & 1018 & $0.5 \pm 0.15$ & 0.4 & 0.7 & - & - & - & RI, MS \\
\hline Limonene & 1025 & 1020 & $1.5 \pm 0.70$ & 0.8 & 2.2 & - & - & - & RI, MS \\
\hline (Z)- $\beta$-Ocimene & 1029 & 1024 & $0.1 \pm 0.06$ & 0.1 & 0.2 & - & - & - & RI, MS \\
\hline (E)-2-Octenal & 1034 & 1034 & - & - & - & $0.4 \pm 0.29$ & 0.1 & 0.8 & RI, MS \\
\hline (E)- $\beta$-Ocimene & 1041 & 1036 & $2.6 \pm 2.21$ & 0.9 & 5.1 & - & - & - & RI, MS \\
\hline$\gamma$-Terpinene & 1051 & 1047 & $1.1 \pm 0.51$ & 0.5 & 1.5 & - & - & - & RI, MS \\
\hline trans-Sabinene hydrate & 1053 & 1050 & $1.0 \pm 0.36$ & 0.7 & 1.4 & - & - & - & RI, MS \\
\hline 1-Octanol & 1063 & 1057 & - & - & - & $6.0 \pm 1.93$ & 2.8 & 8.8 & RI, MS \\
\hline Terpinolene & 1082 & 1078 & $0.1 \pm 0.06$ & 0.1 & 0.2 & - & - & - & RI, MS \\
\hline Nonanal & 1076 & 1081 & - & - & - & $25.8 \pm 10.1$ & 16.5 & 38.2 & RI, MS \\
\hline Linalool & 1086 & 1086 & $17.8 \pm 7.14$ & 9.6 & 22.6 & $1.7 \pm 0.21$ & 1.5 & 1.8 & RI, MS \\
\hline Tetrahydrolinalool & 1099 & 1095 & $4.1 \pm 3.07$ & 0.7 & 6.7 & - & - & - & RI, MS, Ref \\
\hline Dihydrolinalool & 1118 & 1114 & $10.8 \pm 3.50$ & 8.5 & 14.8 & - & - & - & RI, MS, Ref \\
\hline (E)-2-Nonen-1-ol & 1149 & 1153 & - & - & - & $2.2 \pm 1.65$ & 0.6 & 4.6 & RI, MS \\
\hline 1-Phenylethyl acetate & 1166 & 1163 & - & - & - & $0.1 \pm 0.05$ & 0.1 & 0.2 & RI, MS \\
\hline Terpinen-4-ol & 1164 & 1164 & $0.3 \pm 0.20$ & 0.1 & 0.5 & - & - & - & RI, MS \\
\hline$\alpha$-Terpineol & 1176 & 1173 & $\operatorname{tr}$ & $\operatorname{tr}$ & $\operatorname{tr}$ & - & - & - & RI, MS \\
\hline Decanal & 1180 & 1182 & - & - & - & $1.6 \pm 0.74$ & 0.7 & 2.5 & RI, MS \\
\hline Undecanal & 1285 & 1285 & - & - & - & $1.0 \pm 1.03$ & 0.2 & 2.8 & RI, MS \\
\hline Methyl anthranilate & 1308 & 1302 & $0.2 \pm 0.10$ & 0.1 & 0.3 & - & - & - & RI, MS \\
\hline (E)-Jasmone & 1356 & 1360 & $\operatorname{tr}$ & $\operatorname{tr}$ & $\operatorname{tr}$ & - & - & - & RI, MS \\
\hline Isocaryophyllene & 1409 & 1405 & $\operatorname{tr}$ & $\operatorname{tr}$ & $\operatorname{tr}$ & - & - & - & RI, MS \\
\hline
\end{tabular}


Table 4. Cont.

\begin{tabular}{|c|c|c|c|c|c|c|c|c|c|}
\hline \multirow{2}{*}{ Components $^{\text {a }}$} & \multirow{2}{*}{ RI(Lit) ${ }^{b}$} & \multirow{2}{*}{$\mathbf{R I}^{\mathrm{c}}$} & \multicolumn{3}{|c|}{ Clementine flower $^{\mathrm{d}}$} & \multicolumn{3}{|c|}{ Asphodel Flower ${ }^{\text {e }}$} & \multirow{2}{*}{ Identification ${ }^{\mathrm{g}}$} \\
\hline & & & Mean $\pm \operatorname{SD}^{f}$ & Min. & Max. & Mean \pm SD $^{\mathrm{f}}$ & Min. & Max. & \\
\hline$(E)$ - $\beta$-Farnesene & 1446 & 1442 & $\operatorname{tr}$ & $\operatorname{tr}$ & $\operatorname{tr}$ & - & - & - & RI, MS \\
\hline$(E, E)-\alpha$-Farnesene & 1498 & 1492 & $0.1 \pm 0.00$ & 0.1 & 0.1 & - & - & - & RI, MS \\
\hline$(E)$-Nerolidol & 1553 & 1548 & $\operatorname{tr}$ & $\operatorname{tr}$ & $\operatorname{tr}$ & - & - & - & RI, MS \\
\hline Total identification (\%) & & & $81.2 \pm 5.75$ & 75.5 & 87.0 & $85.9 \pm 2.66$ & 82.4 & 90.1 & \\
\hline Hydrocarbons & & & $48.0 \pm 8.16$ & 39.7 & 56.0 & - & - & - & \\
\hline Oxygenated compounds & & & $33.1 \pm 11.88$ & 19.5 & 41.3 & $85.9 \pm 2.66$ & 82.4 & 90.1 & \\
\hline Phenolic compounds & & & $0.2 \pm 0.1$ & 0.1 & 0.3 & $3.7 \pm 0.99$ & 1.8 & 4.5 & \\
\hline Furan compounds & & & - & - & - & $8.2 \pm 4.42$ & 4.3 & 16.7 & \\
\hline Terpenic compounds & & & $80.8 \pm 5.75$ & 75.1 & 86.6 & $0.8 \pm 0.85$ & 0.1 & 1.9 & \\
\hline Ketones & & & $\operatorname{tr}$ & - & $\operatorname{tr}$ & - & - & - & \\
\hline Aldehydes & & & - & - & - & $43.8 \pm 13.47$ & 31.2 & 62.8 & \\
\hline Esters & & & $0.2 \pm 0.1$ & 0.1 & 0.3 & $23.2 \pm 14.58$ & 5.8 & 43.1 & \\
\hline Alcohols & & & $32.9 \pm 11.8$ & 19.4 & 41.1 & $11 \pm 4.13$ & 4.3 & 17 & \\
\hline
\end{tabular}

\footnotetext{
a Order of elution is given on apolar coloumn (Rtx-1). ${ }^{b}$ Retention indice of literature on the apolar column reported from references [27,28]. ${ }^{\mathrm{c}}$ Retention indice on the Rtx-1 apolar column. ${ }^{\mathrm{d}}$ Six clementine flower specimens were collected from Corsica oriental plain. ${ }^{\mathrm{e}}$ Six Asphodele flower specimens were collected from six localities of Corsica. ${ }^{\mathrm{f}}$ Means \pm SD, Min. and Max. values expressed as percentages; tr trace $(<0.05 \%),{ }^{\mathrm{g}} \mathrm{RI}$, Retention indice; MS, mass spectra in electronic impact mode. Ref., compounds identified from commercial data libraries: Konig et al. [27](Samples 8, 34 and 35) and NIST [28] (Samples 3 and 11).
}

The 23 "not-clementine" honey samples (group II) were dominated by phenolic compounds (23.2\%-60.4\%) followed by linear compounds $(11.3 \%-53.4 \%)$. The main compounds were phenylacetaldehyde C14 (3.7\%-36.2\%), benzaldehyde C9 (2.5\%-18.4\%) and methyl-benzene C2 $(1.5 \%-17.3 \%)$. Furanic compounds (average: $7.5 \%$ ) were less abundant than in "clementine" honeys (average: $26.2 \%$ ), and acid components (average: 10.3\%) were more abundant than in the "clementine" honeys (average: 6.8\%). To our knowledge, only one previous report focused on the volatile fraction of asphodel unifloral honeys from Sardinia [18]. Methyl syringate was detected in asphodel nectar in high concentrations and was therefore considered a marker of asphodel honeys [19]. A low content of this component (C42: $0.1 \%-4.1 \%)$ was reported in the volatile fraction of "spring" honey samples (18-21, 24-30, 32, 33 and 36-41). Additionally, the amount of methyl syringate was unrelated to the presence of Asphodelus pollen in the pollen spectrum. This result could be explained by the extreme "under-represented" type of Asphodelus pollen in Corsican "spring" honeys and/or by other nectar contributions in these honeys. The sample 18 exhibited the association of Citrus sp. and A. sinensis; it was grouped with the "not-clementine" honey. In this sample, the citrus nectar contribution was less important than in "clementine" honeys in accordance with the lower concentrations of lilac aldehyde isomers.

To our knowledge, the volatile composition of $A$. ramosus subsp. ramosus flowers is reported here for the first time (Table 4). The HS-SPME volatile fraction of asphodel flowers was dominated by oxygenated compounds, especially linear compounds. Nonanal (16.5\%-38.2\%), (Z)-3-hexenyl acetate $(5.2 \%-41.8 \%)$, octanal $(3.5 \%-12.6 \%), 1$-octanol $(5.7 \%-8.8 \%)$ and heptanal $(3.1 \%-9.5 \%)$ were 
identified as major compounds. The two main components of the honey volatile fraction (phenylacetaldehyde and benzaldehyde) were detected in low concentrations in the flowers. Moreover, methyl syringate (a marker of asphodel honey) was not detected in the flowers analyzed. This result showed that a direct relationship between the volatile fractions of asphodel flowers and the corresponding "spring" honeys could not be established using HS-SPME analysis.

Finally, the characteristic compounds of the volatile fraction of Corsican "chestnut grove" (acetophenone and 2-aminoacetophenone) [24] and "Erica arborea spring maquis" ( $p$-anisaldehyde and 4-propylanisol) honeys [29] were found in low concentrations or not detected in the "spring" honeys studied.

\subsection{Correlation of Melissopalynological and Chemical Data}

To identify relationships between the melissopalynological analysis and volatile composition data of honey samples, CCA was applied on the matrix linked the relative amounts of the 17 volatile compounds (previously used in section "Chemical variability of Corsican "spring" honeys") and the relative frequency (explanatory variables) of eight nectariferous taxa (T7-T9, T11, T13, T14, T18 and T22).

The correlations between the volatile composition and melissopalynolgical data were show in Figure 2. The first CCA axis was negatively related Trifolium sp. T9, Prunus form T11, Echium sp. T13 and Citrus sp. T18 to methyl-benzene C2, 2,2,4,6,6-pentametylheptane C12, three lilac aldehydes (C25, C27 and C28), two $p$-menth-1-en-9-al isomers (C31 and C32), methyl antranilate $\mathbf{C 3 8}$ and $c i s-p$-mentha-1(7),8-dien-1-hydroperoxide C39. The second axis negatively related Trifolium sp. T9 and Asphodelus T22 to methyl-benzene C2, 3-furaldehyde C5, Benzaldehyde C9, 2,2,4,6,6-pentametylheptane C12, phenlyacetaldehyde $\mathbf{C 1 4}$ and methyl syringate $\mathbf{C 4 2}$.

The sample distribution showed the occurrence of two main groups, group I (17 samples: 1-17) and group II (24 samples: 18-41), which correspond to the groups defined in "Determination of geographical and botanical origins of Corsican "spring" honeys" Group I was characterized not only by the significant presence of lilac aldehyde isomers (C25, C27 and C28), $p$-menth-1-en-9-al isomers (C31 and C32) and methyl anthranilate C38, but also by the high abundance of taxa: Citrus sp. T18, Echium sp. T13 and Prunus form T11 (group I: 6.3\%, 9.5\% and 3.8\% versus group II: $0.1 \%$, 4.9\% and $0.8 \%$, respectively). According to the literature [15,16,30-33], all these compounds had been considered as characteristic components of citrus honey. From these results, it appeared that the other nectariferous taxa Echium sp. and Prunus form displayed a polleniferous role in these honey samples.

Group II included 24 samples that had great diversity. According to the sample distribution, we could distinguish 20 honey samples (18-34, 37, 40 and 41), which had higher values of phenylacetaldehyde C14 and methyl syringate C38 (21.4\% and 0.7\%, respectively). These honeys were also characterized by numerous herbaceous taxa with potential for nectar contribution, such as Lotus sp. T7, Salix sp. T8, Apiaceae T14 and Asphodelus T22 (3.5\%, 4.4\%, 3.2\% and 0.6\% versus I: $2.0 \%, 4.6 \%, 0.4 \%$ and $0.03 \%$, respectively). As previously reported in literature data [19], the nectar contribution of Asphodelus T22 in these honey samples was characterized by the presence of methyl syringate C38. In the same way, phenylacetaldehyde C14 was reported as main volatile compound of 
Salix honeys [35] and Asphodelus honey [18]. For the other nectariferous species Lotus sp. T7 and Apiaceae T14, no chemical markers of nectar contribution was reported in previous studies.

Figure 2. Correlation between melissopalynological and volatile data of "spring" honey by canonical correspondence analysis (CCA).

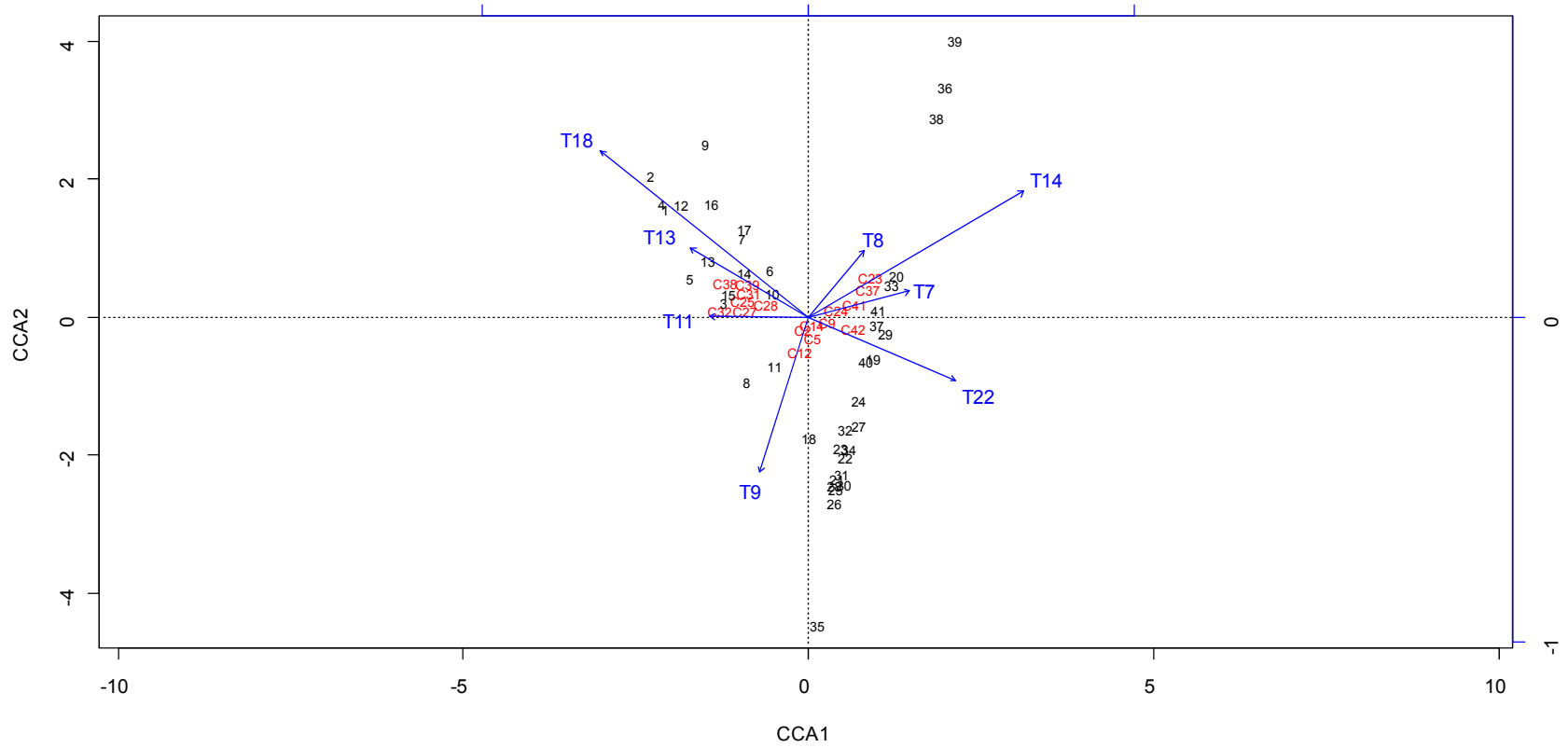

Variables: taxa number corresponding to those of Tables 2 and 3; volatile components number corresponding to those of Table 3. In the CCA plot, location of each sample indicated its compositional similarity to each other; volatile components locations indicated the similarity of their distribution to each other; length of taxa indicated the importance to the ordination, and the direction of taxa vector indicated its correlation with each axes. The perpendiculars drawn from volatile components to taxa give approximate ranking of volatile components response to the taxa variables.

\section{Conclusions}

Corsican "spring" honeys can be classified into two categories according to melissopalynological analysis: (1) honeys characterized by the association of cultivated plants, especially $C$. sinensis $\times$ reticulata with other Citrus species, A. sinensis and other fruit trees; (2) honeys without cultivated taxa, but with herbaceous species (A. ramosus subsp. ramosus, Trifolium sp., Echium sp., Apiaceae, Brassicaceae, Lotus sp., etc.), low shrub species (Rubus sp. and Lavandula stoechas) and some polleniferous taxa with precocious flowering (P. lentiscus and Phillyrea sp.).

Analysis of the volatile fraction of "spring" honeys also demonstrated the existence of two main groups in this range. The volatile fractions were often characterized by high amounts of phenylacetaldehyde, benzaldehyde and methyl-benzene. However, the chemical composition of "clementine" honeys was dominated by three lilac aldehyde isomers that were absent in the "not-clementine" honeys. The statistical analysis showed clearly that the "clementine" honeys were characterized by high volatile content (total peak area), methyl anthranilate, lilac aldehydes, $p$-menth-1-en-9-al isomers and some cultivated taxa, while the "not-clementine" honeys were characterized by phenylacetaldehyde, methyl syringate and complex taxa associations. The richness of 
linalool derivatives in the volatile fraction of clementine flowers suggested biochemical transformation occurring during honeybee activity or honey conservation in the hive.

Finally, it appeared that melissopalynological analysis was necessary for the certification of geographical origin and was useful for the determination of botanical origin. Moreover, analysis of the volatile composition could be used to specify the characteristics of volatile compounds in relation to the predominance and/or complexity of botanical origins of the product, especially when nectariferous species have an "under-represented" pollen type in the pollen spectrum, such as Citrus sp. or Asphodelus sp.

\section{Acknowledgments}

The authors are indebted to the Délégation Régionale à la Recherche et à la Technologie de Corse (DRRT), the Collectivité Territoriale de Corse (CTC) and European Community for partial financial support.

\section{Conflicts of Interest}

The authors declare no conflict of interest.

\section{References}

1. Battesti, M.J.; Gamisans, J.; Piana, L. Définition du Périmètre de Production-Rapport des Experts en Vue de la Mise à l'Enquête. Demande de Reconnaissance en A.O.C. <Miel de Corse-Mele di Corsica>; Institut National des Appelations d'Origine (INAO): Corte, France, 1997.

2. Décret $\mathrm{n}^{\circ}$ 2010-1045 du 31 Août 2010 Relatif à l'Appellation d'Origine Contrôlée $<$ Miel de Corse-Mele di Corsica $>$. Avaiable online: http://www.legifrance.gouv.fr/ affichTexte.do?cidTexte=JORFTEXT000022783277 (accessed on 1 September 2013).

3. Council Regulation (EC) No 510/2006 on the Protection of Geographical Indications and Designations of Origin for Agricultural Products and Foodstuffs. "Miel de Corse/Mele di Corsica". EC No: FR-PDO-0105-0066-20.04.2011. Available online: http://eur-lex.europa.eu/LexUriServ/ LexUriServ.do?uri=OJ:C:2013:134:0039:0048:EN:PDF (accessed on 1 September 2013).

4. Piana, L.; Persano Oddo, L.; Bentabol, A.; Bruneau, E.; Bogdanov, S.; Guyot Declerck, C. Sensory analysis applied to honey: State of the art. Apidologie 2004, 35, $26-27$.

5. Battesti, M.J. Contribution à la Melissopalynologie Méditerranéenne: Les Miels Corses. Ph.D. Thesis, University of Marseille St. Jérôme, Marseille, France, 1990.

6. Persano Oddo, L.; Piana, L.; Bogdanov, S.; Bentabol, A.; Gotsiou, P.; Kerkvliet, J. Botanical species giving unifloral honey in Europe. Apidologie 2004, 26, 82-93.

7. Persano Oddo, L.; Piro, R. Main European unifloral honeys: Descriptive sheets. Apidologie 2004, $35,38-81$.

8. Persano Oddo, L.; Sabatini, A.G.; Accorti, M.; Colombo, R.; Marcazzan, G.L.; Piana, L.; Piazza, M.G.; Pulcini, P. I Mieli Uniflorali Italiani-Nuove Schede di Caratterizzazione; Ministero delle Politiche Agricole e Forestali: Gradoli, Italy, 2000. 
9. Floris, I.; Palmieri, N.; Satta, A. Caratteristiche Melissopalinologiche dei Mieli di Sardegna. In I Mieli Regionali Italiani-Caratterizzazion Melissopalinologica; Ministero delle Politiche Agricole Alimentari e Forestali \& C.R.A. Istituto Sperimentale per la Zoologia Agraria, Sezione di Apicoltura: Roma, Italy, 2007.

10. Jeanmonod, D.; Gamisans, J. Flora Corsica; Edisud: Aix-en-Provence, France, 2007.

11. Battesti, M.J.; Goeury, C. Efficacité de l'analyse mélitopalynologique quantitative pour la certification des origines géographique et botanique des miels: Le modèle des miels corses. Rev. Palaeobot. Palynol. 1992, 75, 77-102.

12. Von Der Ohe, W.; Persano Oddo, L.; Piana, M.L.; Morlot, M.; Martin, P. Harmonized methods of melissopalynology. Apidologie 2004, 35, 18-23.

13. Cuevas-Glory, L.F.; Pino, J.A.; Santiago, L.S.; Sauri-Duch, E. A review of volatile analytical methods for determining the botanical origin of honey. Food Chem. 2007, 103, 1032-1043.

14. Sesta, G.; Piana, M.L.; Persano Oddo, L.; Lusco, L.; Belligoli, P. Methyl anthranilate in Citrus honey. Analytical methode and suitability as a chemical marker. Apidologie 2008, 39, 334-342.

15. Alissandrakis, E.; Tarantilis, P.A.; Harizanis, P.C.; Polissiou, M. Aroma investigation of unifloral Greek citrus honey using solid-phase microextraction coupled go gas chromatographic-mass spectrometric analysis. Food Chem. 2007, 100, 396-404.

16. Alissandrakis, E.; Daferera, D.; Tarantilis, P.A.; Polissiou, M.; Harizanis, P.C. Ultrasound-assisted extraction of volatile compounds from citrus flowers and citrus honey. Food Chem. 2003, 82, 575-582.

17. Ferreres F.; Giner, J.M., Tomas-Barberan, F.A. A comparative study of hesperetin and methyl anthranilate as markers of the floral origin of citrus honey. J. Sci. Food Agric. 1994, 65, 371-372.

18. Jerkovic, I.; Tuberoso, C.I.G.; Kasum, A.; Marijanovic, Z. Volatile compounds of Asphodelus microcarpus Salzm. et Viv. Honey obtained by HS-SPME and USE analyzed by GC/MS. Chem. Biodivers. 2011, 8, 587-598.

19. Tuberoso, C.I.G.; Bifulco, E.; Jerkovic, I.; Caboni, P.; Cabras, P.; Floris, I. Methyl syringate: A chemical marker of asphodel (Asphodelus microcarpus Salzm. et Viv.) monofloral honey. J. Agric. Food Chem. 2009, 57, 3895-3900.

20. Donarski, J.A.; Jones, S.A.; Charlton, A.J. Application of cryoprobe ${ }^{1} \mathrm{H}$ Nuclear Magnetic Resonance spectroscopy and multivariate analysis for the verification of Corsican honey. J. Agric. Food Chem. 2008, 56, 5451-5456.

21. Woodcock, T.; Downey, G.; O’Donnell, C. Near infrared spectral fingerprinting for confirmation of claimed PDO provenance of honey. Food Chem. 2009, 114, 742-746.

22. Stanimirova, I.; Üstün, B.; Cajka, T.; Riddelova, K.; Hajslova, J.; Buydens, L.M.C. Tracing the geographical origin of honeys based on volatile compounds profiles assessment using pattern recognition techniques. Food Chem. 2010, 118, 171-176.

23. Gonnet, M., Vache, G. Le Goût du Miel; U.N.A.F.: Paris, France, 1985.

24. Yang, Y.; Battesti, M.J.; Djabou, N.; Muselli, A.; Paolini, J.; Tomi, P.; Costa, J. Melissopalynological origin determination and volatile composition analysis of Corsican "chestnut grove" honeys. Food Chem. 2012, 132, 2144-2154.

25. Aubert, S.; Gonnet, M. Mesure de la couleur des miels. Apidologie 1983, 14, 105-118.

26. Bogdanov, S. Charakterisierung von Schweizer Sortenhonigen. Agrarforschung 1997, 4, 427-430. 
27. Konig, W.A.; Hochmuth, D.H.; Joulain, D. Terpenoids and Related Constituents of Essential oils; Library of Mass Finder 2.1, Institute of Organic Chemistry: Hamburg, Germany, 2001.

28. National Institute of Standards and Technology (NIST). Spectral Database for Organic Compounds. In NIST Chemistry WebBook; Available online: http://webbook.nist.gov/chemistry (accessed on 1 September 2013).

29. Yang, Y.; Battesti, M.J.; Paolini, J.; Muselli, A.; Tomi, P.; Costa, J. Melissopalynological origin determination and volatile composition analysis of Corsican "Erica arborea spring maquis" honeys. Food Chem. 2012, 134, 37-47.

30. Perez, R.A.; Sanchez-Brunete, C.; Calvo, R.M.; Tadeo, J.L. Analysis of volatiles from Spanish honeys by solid-phase microextraction and gas chromatography-mass spectrometry. J. Agric. Food Chem. 2002, 50, 2633-2637.

31. Castro-Vazquez, L.; Diaz-Maroto, M.C.; Perez-Coello, M.S. Aroma composition and new chemical markers of Spanish citrus honeys. Food Chem. 2007, 103, 601-606.

32. Castro-Vazquez, L.; Diaz-Maroto, M.C.; Gonzalez-Vinas, M.A.; Perez-Coello, M.S. Differentiation of monofloral citrus, rosemary, eucalyptus, lavender, thyme, and heather honeys based on volatile composition and sensory descriptive analysis. Food Chem. 2009, 112, 1022-1030.

33. Aliferis, K.A.; Tarantilis, P.A.; Harizanis, P.C.; Alissandrakis E. Botanical discrimination and classification of honey samples applying gas chromatography/mass spectrometry fingerprinting of headspace volatile compounds. Food Chem. 2010, 121, 856-862.

34. Maurizio, A.; Louveaux, J. Pollens de Plantes Mellifères d'Europe; Union des Groupements Apicoles Français: Paris, France, 1965.

35. De la Fuente, E.; Sanz, M.L.; Martinez-Castro, I.; Sanz, J.; Ruiz-Matute, A.I. Volatile and carbohydrate composition of rare unifloral honeys from Spain. Food Chem. 2007, 105, 84-93.

(C) 2014 by the authors; licensee MDPI, Basel, Switzerland. This article is an open access article distributed under the terms and conditions of the Creative Commons Attribution license (http://creativecommons.org/licenses/by/3.0/). 